\title{
Cobordisms of maps with singularities of given class
}

\author{
YOSHIFUMI ANDO
}

\begin{abstract}
Let $P$ be a smooth manifold of dimension $p$. We will describe the group of all cobordism classes of smooth maps of $n$-dimensional closed manifolds into $P$ with singularities of given class (including all fold singularities if $n \geqq p$ ) in terms of certain stable homotopy groups by applying the homotopy principle on the existence level, which is assumed to hold for those smooth maps. It will enable us to construct an explicit classifying space for this cobordism group in the dimensions $n<p$ and $n \geqq p \geqq 2$.
\end{abstract}

57R45; 57R90, 58A20

\section{Dedicated to Professor Takao Matumoto on his sixtieth birthday}

\section{Introduction}

Let $N$ and $P$ be smooth $\left(C^{\infty}\right)$ manifolds of dimensions $n$ and $p$ respectively. Let $k \gg n, p(k$ may be $\infty)$. Denote by $J^{k}(N, P)$ the $k$-jet bundle of the manifolds $N$ and $P$ with the canonical projection $\pi_{N}^{k} \times \pi_{P}^{k}$ onto $N \times P$ and the fiber $J^{k}(n, p)$ of all $k$-jets of smooth map germs $\left(\mathbb{R}^{n}, 0\right) \rightarrow\left(\mathbb{R}^{p}, 0\right)$. Here, $\pi_{N}^{k}$ and $\pi_{P}^{k}$ map a $k$-jet to its source and target respectively. Let $\Omega=\Omega(n, p)$ denote a nonempty open subspace of $J^{k}(n, p)$ which is invariant with respect to the action of $L^{k}(p) \times L^{k}(n)$, where $L^{k}(m)$ denotes the group of $k$-jets of germs of diffeomorphisms of $\left(\mathbb{R}^{m}, 0\right)$. Let $\Omega(N, P)$ denote the open subbundle of $J^{k}(N, P)$ associated to $\Omega(n, p)$. A smooth map $f: N \rightarrow P$ is called an $\Omega$-regular map if and only if $j^{k} f(N) \subset \Omega(N, P)$.

Let $C_{\Omega}^{\infty}(N, P)$ denote the space of all $\Omega$-regular maps of $N$ to $P$ equipped with the $C^{\infty}$ topology. Denote by $\Gamma_{\Omega}(N, P)$ the space consisting of all continuous sections of the fiber bundle $\pi_{N}^{k} \mid \Omega(N, P): \Omega(N, P) \rightarrow N$ equipped with the compact-open topology. Then we have the continuous map $j_{\Omega}: C_{\Omega}^{\infty}(N, P) \rightarrow \Gamma_{\Omega}(N, P)$ defined by $j_{\Omega}(f)=j^{k} f$. We say that $\Omega(n, p)$ satisfies the homotopy principle (simply h-principle) if any section $s$ in $\Gamma_{\Omega}(N, P)$ has an $\Omega$-regular map $f$ such that $j^{k} f$ is homotopic to $s$ as sections. In this paper we say that $\Omega(n, p)$ satisfies the relative homotopy principle on the existence level if the following property (h-P) holds. 
(h-P) Let $C$ be a closed subset of $N$. Let $s$ be a section of $\Gamma_{\Omega}(N, P)$ which has an $\Omega$-regular map $g$ defined on a neighborhood of $C$ into $P$, where $j^{k} g=s$. Then there exists an $\Omega$-regular map $f: N \rightarrow P$ such that $j^{k} f$ is homotopic to $s$ relative to a neighborhood of $C$ by a homotopy $s_{\lambda}$ in $\Gamma_{\Omega}(N, P)$ with $s_{0}=s$ and $s_{1}=j^{k} f$.

Let $i_{+m}: J^{k}(n, p) \rightarrow J^{k}(n+m, p+m)$ denote the map defined by $i_{+m}\left(j_{0}^{k} f\right)=$ $j_{0}^{k}\left(f \times \operatorname{id}_{\mathbb{R}^{m}}\right)$ for a positive integer $m$, where $f:\left(\mathbb{R}^{n}, 0\right) \rightarrow\left(\mathbb{R}^{p}, 0\right)$ and $\operatorname{id}_{\mathbb{R}^{m}}$ is the identity of $\mathbb{R}^{m}$. Denote by $\Omega_{\star}=\Omega_{\star}(n+1, p+1)$ a nonempty open subspace of $J^{k}(n+1, p+1)$ which is invariant with respect to the action of $L^{k}(p+1) \times$ $L^{k}(n+1)$ and satisfies $i_{+1}(\Omega(n, p)) \subset \Omega_{\star}(n+1, p+1)$. Let $P$ be a smooth manifold of dimension $p$. We define the notion of $\Omega_{\star}$-cobordisms of $\Omega$-regular maps to $P$. Let $f_{i}: N_{i} \rightarrow P(i=0,1)$ be two $\Omega$-regular maps, where $N_{i}$ are closed smooth $n$-dimensional manifolds. We say that they are $\Omega_{\star}$-cobordant when there exists an $\Omega_{\star}$-regular map, called an $\Omega_{\star}$-cobordism, $\mathfrak{C}:(W, \partial W) \rightarrow(P \times[0,1], P \times 0 \cup P \times 1)$ such that, for a sufficiently small positive real number $\epsilon$ :

(i) $W$ is a compact smooth manifold of dimension $n+1$ with $\partial W$ equal to $N_{0} \cup N_{1}$ and the collar of $\partial W$ is identified with $N_{0} \times[0, \epsilon] \cup N_{1} \times[1-\epsilon, 1]$,

(ii) $\mathfrak{C} \mid N_{0} \times[0, \epsilon]=f_{0} \times \operatorname{id}_{[0, \epsilon]}$ and $\mathfrak{C} \mid N_{1} \times[1-\epsilon, 1]=f_{1} \times \operatorname{id}_{[1-\epsilon, 1]}$.

We similarly define the notion of oriented $\Omega_{\star}$-cobordisms of $\Omega$-regular maps by providing manifolds concerned with orientations, where $N_{0} \cup N_{1}$ in (i) should be replaced by $N_{0} \cup\left(-N_{1}\right)$. Let $\mathfrak{N}\left(n, P ; \Omega, \Omega_{\star}\right)$ (respectively $\mathfrak{O}\left(n, P ; \Omega, \Omega_{\star}\right)$ ) denote the monoid of all $\Omega_{\star}$-cobordism (respectively oriented $\Omega_{\star}$-cobordism) classes of $\Omega$-regular maps to $P$. In this paper we will describe these monoids of cobordism classes in terms of certain stable homotopy groups.

We need some notation for this purpose. Let $\mathcal{E} \rightarrow X$ and $\mathcal{F} \rightarrow Y$ be smooth vector bundles of dimensions $n$ and $p$ over smooth manifolds, and let $\pi_{X}$ and $\pi_{Y}$ be the projections of $X \times Y$ onto $X$ and $Y$ respectively. Define the vector bundle $J^{k}(\mathcal{E}, \mathcal{F})$ over $X \times Y$ by

$$
J^{k}(\mathcal{E}, \mathcal{F})=\bigoplus_{i=1}^{k} \operatorname{Hom}\left(S^{i}\left(\pi_{X}^{*}(\mathcal{E})\right), \pi_{Y}^{*}(\mathcal{F})\right)
$$

with the canonical projections $\pi_{X}^{k}: J^{k}(\mathcal{E}, \mathcal{F}) \rightarrow X$ and $\pi_{Y}^{k}: J^{k}(\mathcal{E}, \mathcal{F}) \rightarrow Y$. Here, $S^{i}(\mathcal{E})$ is the vector bundle $\bigcup_{x \in X} S^{i}\left(\mathcal{E}_{x}\right)$ over $X$, where $S^{i}\left(\mathcal{E}_{x}\right)$ denotes the $i$-fold symmetric product of the fiber $\mathcal{E}_{x}$ over $x$. The canonical fiber

$$
\bigoplus_{i=1} \operatorname{Hom}\left(S^{i}\left(\mathbb{R}^{n}\right), \mathbb{R}^{p}\right)
$$


is canonically identified with $J^{k}(n, p)$. If we provide $N$ and $P$ with Riemannian metrics, then $J^{k}(T N, T P)$ is identified with $J^{k}(N, P)$ over $N \times P$ (see Section 2). Let $\Omega(\mathcal{E}, \mathcal{F})$ denote the open subbundle of $J^{k}(\mathcal{E}, \mathcal{F})$ associated to $\Omega(n, p)$.

Let $G_{m}$ refer to the Grassmann manifold $G_{m, \ell}$ (respectively oriented Grassmann manifold $\widetilde{G}_{m, \ell}$ ) of all $m$-subspaces (respectively oriented $m$-subspaces) of $\mathbb{R}^{\ell+m}$. Let $\gamma_{G_{m}}^{m}$ and $\hat{\gamma}_{G_{m}}^{\ell}$ denote the canonical vector bundles of dimensions $m$ and $\ell$ over the space $G_{m}$ respectively such that $\gamma_{\boldsymbol{G}_{m}}^{m} \oplus \hat{\gamma}_{\boldsymbol{G}_{m}}^{\ell}$ is the trivial bundle $\varepsilon_{\boldsymbol{G}_{m}}^{\ell+m}$. Let $T\left(\hat{\gamma}_{\boldsymbol{G}_{m}}^{\ell}\right)$ denote the Thom space of $\hat{\gamma}_{G_{m}}^{\ell}$. The spaces $\left\{T\left(\hat{\gamma}_{G_{m}}^{\ell}\right)\right\}_{\ell}$ constitute a spectrum. Let

$$
i^{G}: G_{n} \rightarrow G_{n+1}
$$

denote the injection mapping an $n$-plane $a$ to the $(n+1)$-plane generated by $a$ and the $(n+\ell+1)$-st unit vector $\mathbf{e}_{n+\ell+1}$ in $\mathbb{R}^{n+\ell+1}$. Let

$$
\boldsymbol{\Omega}=\Omega\left(\gamma_{G_{n}}^{n}, T P\right) \quad \text { and } \quad \boldsymbol{\Omega}_{*}=\Omega_{\star}\left(\gamma_{G_{n+1}^{n+1}}^{n}, T P \oplus \varepsilon_{P}^{1}\right)
$$

be the open subbundles of $J^{k}\left(\gamma_{G_{n}}^{n}, T P\right)$ and $J^{k}\left(\gamma_{G_{n+1}}^{n+1}, T P \oplus \varepsilon_{P}^{1}\right)$ associated to $\Omega(n, p)$ and $\Omega_{\star}(n+1, p+1)$ respectively, where $\varepsilon_{P}^{1}$ denotes the trivial bundle $P \times \mathbb{R}$. Set

$$
\hat{\gamma}_{\boldsymbol{\Omega}}^{\ell}=\left.\left(\pi_{G_{n}}^{k}\right)^{*}\left(\hat{\gamma}_{G_{n}}^{\ell}\right)\right|_{\boldsymbol{\Omega}} \quad \text { and } \quad \hat{\gamma}_{\mathbf{\Omega}_{*}}^{\ell}=\left.\left(\pi_{G_{n+1}}^{k}\right)^{*}\left(\hat{\gamma}_{G_{n+1}}^{\ell}\right)\right|_{\boldsymbol{\Omega}_{*}} .
$$

There exists a fiberwise map $\Delta^{\left(\Omega, \Omega_{\star}\right)}: \boldsymbol{\Omega} \rightarrow \boldsymbol{\Omega}_{\star}$ associated to

$$
i_{+1} \mid \Omega(n, p): \Omega(n, p) \rightarrow \Omega_{\star}(n+1, p+1)
$$

covering $i^{G} \times \operatorname{id}_{P}$. Then $\Delta^{\left(\Omega, \Omega_{\star}\right)}$ induces the bundle map

$$
\mathbf{b}(\widehat{\gamma})^{\left(\Omega, \Omega_{\star}\right)}: \hat{\gamma}_{\mathbf{\Omega}}^{\ell} \longrightarrow \hat{\gamma}_{\mathbf{\Omega}_{\star}}^{\ell}
$$

covering $\Delta^{\left(\Omega, \Omega_{\star}\right)}$ and the associated map $T\left(\mathbf{b}(\hat{\gamma})^{\left(\Omega, \Omega_{\star}\right)}\right)$ between the Thom spaces. Let $\ell \gg n, p$. We denote the image of

$$
T\left(\mathbf{b}(\hat{\gamma})^{\left(\Omega, \Omega_{\star}\right)}\right)_{*}: \lim _{\ell \rightarrow \infty} \pi_{n+\ell}\left(T\left(\hat{\gamma}_{\mathbf{\Omega}}^{\ell}\right)\right) \longrightarrow \lim _{\ell \rightarrow \infty} \pi_{n+\ell}\left(T\left(\hat{\gamma}_{\mathbf{\Omega}_{\star}}^{\ell}\right)\right)
$$

by $\operatorname{Im}^{\mathfrak{N}}\left(T\left(\mathbf{b}(\widehat{\gamma})^{\left(\Omega, \Omega_{\star}\right)}\right)\right)$ (respectively $\operatorname{Im}^{\mathcal{O}}\left(T\left(\mathbf{b}(\widehat{\gamma})^{\left(\Omega, \Omega_{\star}\right)}\right)\right)$ ) in the unoriented (respectively the oriented) case.

We are ready to state the main result of this paper.

Theorem 1.1 Let $n$ and $p$ be positive integers. Let $P$ be a $p$-dimensional manifold. Let $\Omega(n, p)$ and $\Omega_{\star}(n+1, p+1)$ denote the above open subsets invariant with respect to the actions of $L^{k}(p) \times L^{k}(n)$ and $L^{k}(p+1) \times L^{k}(n+1)$, respectively, such that $i_{+1}(\Omega(n, p)) \subset \Omega_{\star}(n+1, p+1), \Omega(n, p)$ satisfies the $h$-principle and that $\Omega_{\star}(n+1, p+1)$ satisfies the relative $h$-principle on the existence level in $(h-P)$. 
Then there exist isomorphisms

$$
\begin{aligned}
& \mathfrak{n}^{\left(\Omega, \Omega_{\star}\right)}: \mathfrak{N}\left(n, P ; \Omega, \Omega_{\star}\right) \longrightarrow \operatorname{Im}^{\mathfrak{N}}\left(T\left(\mathbf{b}(\widehat{\gamma})^{\left(\Omega, \Omega_{\star}\right)}\right)\right), \\
& \mathfrak{o}^{\left(\Omega, \Omega_{\star}\right)}: \mathfrak{O}\left(n, P ; \Omega, \Omega_{\star}\right) \longrightarrow \operatorname{Im}^{\mathfrak{O}}\left(T\left(\mathbf{b}(\hat{\gamma})^{\left(\Omega, \Omega_{\star}\right)}\right)\right) .
\end{aligned}
$$

Let $\mathcal{K}$ denote the contact group introduced by Mather [37] which acts on $J^{k}(n, p)$ or $J^{k}(n+m, p+m)$. Let $\Omega_{\mathcal{K}}^{m}=\Omega_{\mathcal{K}}(n+m, p+m)$ denote the subset of $J^{k}(n+m, p+m)$ which consists of all $\mathcal{K}$-orbits $\mathcal{K}\left(i_{+m}(z)\right)$ for $k$-jets $z \in \Omega(n, p)$. It will be proved that $\Omega_{\mathcal{K}}(n+m, p+m)$ is an open subset (see Lemma 5.2).

As for the image of $T\left(\mathbf{b}(\widehat{\gamma})^{\left(\Omega, \Omega_{\star}\right)}\right)_{*}$, we will prove the following theorem.

Theorem 1.2 Let $n<p$ and $P$ be as in Theorem 1.1. Let $\Omega(n, p)$ denote a nonempty open subset in $J^{k}(n, p)$ invariant with respect to the action of $\mathcal{K}$ and let $\Omega_{\mathcal{K}}=$ $\Omega_{\mathcal{K}}(n+1, p+1)$ be as above. Then the homomorphism $T\left(\mathbf{b}(\widehat{\gamma})^{\left(\Omega, \Omega_{\mathcal{K}}\right)}\right)_{*}$ in $(1-2)$ is surjective.

Let $\Omega_{\mathcal{K}}\left(\gamma_{G_{n+m}}^{n+m}, \mathbb{R}^{p+m}\right)$ denote the subbundle of $J^{k}\left(\gamma_{G_{n+m}}^{n+m}, \mathbb{R}^{p+m}\right)$ associated to $\Omega_{\mathcal{K}}(n+m, p+m)$. Let $C^{0}(X, Y)$ denote the space consisting of all base-point preserving continuous maps between connected spaces with base points equipped with the compact-open topology. We define the space $B_{\mathfrak{C}}$ by

$$
B_{\mathfrak{C}}=\lim _{m \rightarrow \infty}\left(\operatorname { l i m } _ { \ell \rightarrow \infty } C ^ { 0 } \left(S^{\ell+n-p}, T\left(\left.\left(\pi_{G_{n+m}}^{k}\right)^{*} \hat{\gamma}_{G_{n+m}^{\ell}}\right|_{\left.\left.\Omega_{\mathcal{K}}\left(\gamma_{G_{n+m}}^{n+m}, \mathbb{R}^{p+m}\right)\right)\right)}\right),\right.\right.
$$

where $\mathfrak{C}=\mathfrak{C}\left(n, P ; \Omega, \Omega_{\mathcal{K}}\right)$ means $\mathfrak{N}\left(n, P ; \Omega, \Omega_{\mathcal{K}}\right)$ or $\mathfrak{O}\left(n, P ; \Omega, \Omega_{\mathcal{K}}\right)$ depending on $G_{n+m}=G_{n+m, \ell}$ or $G_{n+m}=\widetilde{G}_{n+m, \ell}$ respectively.

Theorem 1.3 Let $n<p$ or $n \geqq p \geqq 2$. Let $P$ be a closed connected $p$-dimensional manifold such that $P$ is oriented in addition in the case of $\mathcal{O}\left(n, P ; \Omega, \Omega_{\mathcal{K}}\right)$. Let $\Omega(n, p)$ be a nonempty $\mathcal{K}$-invariant open subset such that if $n \geqq p \geqq 2$, then $\Omega(n, p)$ contains all fold jets at least. Then there exist isomorphisms

$$
\begin{aligned}
& \mathfrak{N}\left(n, P ; \Omega, \Omega_{\mathcal{K}}\right) \longrightarrow\left[P, B_{\mathfrak{N}}\right] \\
& \mathfrak{O}\left(n, P ; \Omega, \Omega_{\mathcal{K}}\right) \longrightarrow\left[P, B_{\mathfrak{O}}\right] .
\end{aligned}
$$

In our study of cobordisms of singular maps the h-principle for $\Omega$-regular maps plays a quite important role and our method is available in the dimensions $n \geqq p$ as well. Theorem 1.1 was developed from an observation for fold-maps in the author's paper [5, Theorem 0.4]. Set $\boldsymbol{\Omega}_{\mathcal{K}}^{m}=\Omega_{\mathcal{K}}\left(\gamma_{\boldsymbol{G}_{n+m}}^{n+m}, T P \oplus \varepsilon_{\boldsymbol{P}}^{m}\right)$ and $\hat{\gamma}_{\boldsymbol{\Omega}_{\mathcal{K}}^{m}}^{\ell}=\left.\left(\pi_{\boldsymbol{G}_{n+m}}^{k}\right)^{*}\left(\hat{\gamma}_{\boldsymbol{G}_{n+m}}^{\ell}\right)\right|_{\boldsymbol{\Omega}_{\mathcal{K}}^{m}}$. 
Theorem 1.2 implies that if $n<p$, then $\mathfrak{C}\left(n, P ; \Omega, \Omega_{\mathcal{K}}\right)$ is isomorphic to the stable homotopy group

$$
\lim _{\ell \rightarrow \infty} \pi_{n+\ell}\left(T\left(\hat{\gamma}_{\boldsymbol{\Omega}_{\mathcal{K}}^{\ell}}^{\ell}\right)\right) \approx \lim _{\ell \rightarrow \infty} \pi_{n+\ell}\left(T\left(\hat{\gamma}_{\mathbf{\Omega}_{\mathcal{K}}^{\ell}}^{\ell}\right)\right)
$$

for $m \geqq 1$. If we apply the relative h-principle in Theorem 9.1 to [45, Theorem 9.2] due to Sadykov, then it follows that $\mathfrak{C}\left(n, P ; \Omega, \Omega_{\mathcal{K}}\right)$ is isomorphic to $\pi_{n+\ell}\left(T\left(\hat{\gamma}_{\boldsymbol{\Omega}_{\mathcal{K}}^{\prime}}^{\ell}\right)\right)$ also in the dimensions $n \geqq p \geqq 2$ as well as $n<p$, where $\ell$ and $m$ are sufficiently large integers. By using these stable homotopy groups, we will induce the space $B_{\mathfrak{C}}$ by applying the $S$-duality in Spanier [52] in Section 8. In Section 9 we will see that the h-principle in (h-P) holds for a very wide class of $\mathcal{K}$-invariant open sets $\Omega(n, p)$. Let $\Sigma^{I}(n, p)$ denote the Boardman manifold in $J^{k}(n, p)$ with Boardman symbol $I=\left(i_{1}, \cdots, i_{k}\right)$ defined in [13]. An important example of $\Omega(n, p)$ is the open subset $\Omega^{I}(n, p)$ which is the union of all $\Sigma^{K}(n, p)$ with $K \leqq I$ in the lexicographic order. The results of the paper show the importance of the homotopy type of $\Omega(n, p)$. In $[2 ; 4 ; 5 ; 7]$ we described the homotopy type of $\Omega^{i, 0}(n, p)$ for $i=\max \{n-p+1,1\}$ in terms of orthogonal groups and Stiefel manifolds. Although available only in the case of fold-maps, in Section 10 we will construct a simpler spectrum associated to special generic maps, say definite fold-maps, whose stable homotopy group is a direct summand of $\mathfrak{C}\left(n, P ; \Omega^{i, 0}, \Omega^{i, 0}\right)$ and also construct the corresponding classifying space. This result is a refinement of [5, Theorem 0.3]. We should note that special generic maps do not satisfy the h-principle (see Burlet and de Rham [14] and Saeki and Sakuma [49]). Let $F_{m}$ denote the space of all base point preserving maps of the $m$-sphere $S^{m}$ and let $F=\lim _{m \rightarrow \infty} F_{m}$. When $n=p \geqq 1$ and $P$ is closed and oriented, we will prove that there exists an isomorphism of $\mathcal{O}\left(n, P ; \Omega^{1,0}, \Omega^{1,0}\right)$ onto $[P, F]$, which we gave in $[3 ; 5]$ from a different point of view. Therefore, we may assert that the topology of $B_{\mathfrak{O}}$ will be important in connection with the canonical homomorphism of $\mathfrak{O}\left(n, S^{n} ; \Omega^{1,0}, \Omega^{1,0}\right)$ to $\mathfrak{O}\left(n, S^{n} ; \Omega, \Omega_{\mathcal{K}}\right)$ and the map $F \rightarrow B_{\mathfrak{O}}$ (see (10-8)). We should refer to Chess [16], in which $\mathfrak{O}\left(n, \mathbb{R}^{n} ; \Omega^{1,0}, \Omega^{1,0}\right) \approx \pi_{n}^{s}$ was proved.

The study of h-principles for $\Omega$-regular maps has a long history. Here, we only refer the reader to Smale [51], Hirsch [25], Phillips [40], Feit [21], Gromov [22; 24], Eliashberg [18; 19] and du Plessis [41; 42; 43] for details and further references.

In [20] Eliashberg studied the cobordisms of the solutions of the first order differential relations such as Lagrange and Legendre immersions by applying the h-principles in Gromov [23; 24] and Lees [34]. Sadykov [45] studied and expressed the cobordism group of $\Omega$-regular maps in terms of the stable homotopy group as explained above under the assumption of the relative h-principle in a formal approach. 
In [44] Rimányi and Szúcs constructed a certain classifying space such that the group of cobordism classes of smooth maps of $n$-dimensional manifolds into $P$ having only a given class of $C^{\infty}$ stable singularities is described by the homotopy classes of $P$ to this space in the case $n<p$. In [56] Szúcs developed further results concerning the classifying space and the structure of this cobordism group. The method of construction of this classifying space is quite different from ours of the space $B_{\mathfrak{O}}$ using h-principles in this paper. The results Propositions 67 and 71 in [56] resemble Theorems 1.2 and 1.3 in the corresponding case. In $[27 ; 28 ; 30]$, Kalmár studied the cobordism groups of fold-maps in negative codimensions.

As another line of investigation of cobordisms of singular maps in which h-principles are not available, we refer to Saeki [47], Ikegami and Saeki [26], Saeki [48], Kalmár [29] and Sadykov [46].

We will define the homomorphisms $\mathfrak{n}^{\left(\Omega, \Omega_{\star}\right)}$ and $\mathfrak{o}^{\left(\Omega, \Omega_{\star}\right)}$ in Section 3. In Section 4 we will prove Theorem 1.1. In Section 5 we will prove that $\Omega_{\mathcal{K}}^{m}$ is an open subset. In Section 6 we will prepare several results which are necessary in the proofs of Theorems 1.2 and 1.3. In Section 7 we will prove Theorem 1.2. In Section 8 we will explain how the space $B_{\mathfrak{C}}$ is introduced. In Section 9 we will give a wide class of open sets $\Omega(n, p)$ which satisfy the h-principle in (h-P). Theorem 1.3 will be proved in this section. In Section 10 we will construct a simpler spectrum associated to the open subspace consisting of all regular jets and all definite fold jets in $\Omega^{n-p+1,0}(n, p)$ and the corresponding classifying space. We will show that it yields a direct summand of the cobordism group for fold-maps by using the homotopy type of $\Omega^{n-p+1,0}(n, p)$ and the relative h-principle.

\section{Preliminaries}

Given a fiber bundle $\pi^{\mathcal{E}}: \mathcal{E} \rightarrow X$ and a subset $C$ in $X$, we denote $\left(\pi^{\mathcal{E}}\right)^{-1}(C)$ by $\left.\mathcal{E}\right|_{C}$. Let $\pi^{\mathcal{F}}: \mathcal{F} \rightarrow Y$ be another fiber bundle. A map $\tilde{b}: \mathcal{E} \rightarrow \mathcal{F}$ is called a fiber map covering a map $b: X \rightarrow Y$ if $\pi^{\mathcal{F}} \circ \widetilde{b}=b \circ \pi^{\mathcal{E}}$ holds. The restriction $\widetilde{b}\left|\left(\left.\mathcal{E}\right|_{C}\right): \mathcal{E}\right|_{C} \rightarrow \mathcal{F}$ (or $\left.\mathcal{F}\right|_{b(C)}$ ) is denoted by $\left.\widetilde{b}\right|_{C}$. In particular, for a point $x \in X,\left.\mathcal{E}\right|_{x}$ and $\left.\widetilde{b}\right|_{x}$ are simply denoted by $\mathcal{E}_{x}$ and $\tilde{b}_{x}: \mathcal{E}_{x} \rightarrow \mathcal{F}_{b(x)}$ respectively. The trivial bundle $X \times \mathbb{R}^{\ell}$ is denoted by $\varepsilon_{X}^{\ell}$ (see Steenrod [54]).

Let $\mathcal{E} \rightarrow X$ and $\mathcal{F} \rightarrow Y$ be vector bundles of dimensions $n$ and $p$ respectively. The origin of $\mathbb{R}^{m}$ is simply denoted by 0 for any natural number $m$. We define the action of $L^{k}(p) \times L^{k}(n)$ on $J^{k}(n, p)$ by $\left(j_{0}^{k} h_{1}, j_{0}^{k} h_{2}\right) \cdot j_{0}^{k} f=j_{0}^{k}\left(h_{1} \circ f \circ h_{2}^{-1}\right)$. Let $\mathcal{E}_{1} \rightarrow X_{1}$ and $\mathcal{F}_{1} \rightarrow Y_{1}$ be other vector bundles of dimensions $n$ and $p$ respectively, and let $\tilde{b}_{1}: \mathcal{E} \rightarrow \mathcal{E}_{1}$ and $\tilde{b}_{2}: \mathcal{F} \rightarrow \mathcal{F}_{1}$ be bundle maps covering $b_{1}: X \rightarrow X_{1}$ and $b_{2}: Y \rightarrow Y_{1}$ 
respectively. Then $\tilde{b}_{1}$ and $\tilde{b}_{2}$ yield the isomorphisms $S^{i}\left(\mathcal{E}_{x}\right) \rightarrow S^{i}\left(\mathcal{E}_{1, b_{1}(x)}\right)$ and $\mathcal{F}_{y} \rightarrow \mathcal{F}_{1, b_{2}(y)}$ for any $x \in X$ and $y \in Y$ for $1 \leqq i \leqq k$ and hence, we have the bundle map

$$
\mathbf{j}\left(\tilde{b}_{1}, \tilde{b}_{2}\right): J^{k}(\mathcal{E}, \mathcal{F}) \rightarrow J^{k}\left(\mathcal{E}_{1}, \mathcal{F}_{1}\right)
$$

covering $b_{1} \times b_{2}$. Then $\mathbf{j}\left(\tilde{b}_{1}, \tilde{b}_{2}\right)$ induces the bundle map $\mathbf{j}\left(\tilde{b}_{1}, \tilde{b}_{2}\right)_{\Omega}: \Omega(\mathcal{E}, \mathcal{F}) \rightarrow$ $\Omega\left(\mathcal{E}_{1}, \mathcal{F}_{1}\right)$.

If we provide $N$ and $P$ with Riemannian metrics, then the Levi-Civita connections induce the exponential maps $\exp _{N, x}: T_{x} N \rightarrow N$ and $\exp _{P, y}: T_{y} P \rightarrow P$ for $x \in N$ and $y \in P$ respectively. In dealing with the exponential maps we always consider the convex neighborhoods. We define the smooth bundle map

$$
J^{k}(N, P) \rightarrow J^{k}(T N, T P) \quad \text { over } N \times P
$$

by sending $z=j_{x}^{k} f \in\left(\pi_{N}^{k} \times \pi_{P}^{k}\right)^{-1}(x, y)$ to the $k$-jet of $\left(\exp _{P, y}\right)^{-1} \circ f \circ \exp _{N, x}$ at $\mathbf{0} \in T_{x} N$, which is regarded as an element of $J^{k}\left(T_{x} N, T_{y} P\right)\left(=J_{x, y}^{k}(T N, T P)\right)$ (see Kobayashi and Nomizu [33, Proposition 8.1] for the smoothness of exponential maps). More strictly, $(2-1)$ gives a smooth equivalence of the fiber bundles under the structure group $L^{k}(p) \times L^{k}(n)$. Namely, it gives a smooth reduction of the structure group $L^{k}(p) \times L^{k}(n)$ of $J^{k}(N, P)$ to $O(p) \times O(n)$, which is the structure group of $J^{k}(T N, T P)$. Let $\Sigma^{I}(N, P)$ denote the Boardman manifold in $J^{k}(N, P)$, where $I=\left(i_{1}, \cdots, i_{k}\right)$ is a Boardman symbol such that $n \geqq i_{1} \geqq \cdots \geqq i_{k} \geqq 0$ (see Boardman [13], Levine [35] and Mather [39]). Let $\Omega^{I}(N, P)$ denote the open subset which is the union of all $\Sigma^{K}(N, P)$ with $K \leqq I$ in the lexicographic order. Let $\Sigma^{I}(T N, T P)$ and $\Omega^{I}(T N, T P)$ be the subbundles of $J^{k}(T N, T P)$ associated to $\Sigma^{I}(n, p)$ and $\Omega^{I}(n, p)$, which are identified with $\Sigma^{I}(N, P)$ and $\Omega^{I}(N, P)$ under $(2-1)$, respectively.

Let $f: X \rightarrow Y$ be a continuous map. If $f_{*}: \pi_{i}(X) \rightarrow \pi_{i}(Y)$ is an isomorphism for $0 \leqq i<m$ and an epimorphism for $i=m$, then we call $f$ a homotopy $m$-equivalence in this paper.

\section{Homomorphisms in Theorem 1.1}

Let $\Omega(n, p)$ and $\Omega_{\star}(n+1, p+1)$ be open subsets given in Theorem 1.1.

As usual we provide $\mathfrak{N}\left(n, P ; \Omega, \Omega_{\star}\right)$ and $\mathfrak{O}\left(n, P ; \Omega, \Omega_{\star}\right)$ with the structures of monoids. Namely, given two $\Omega$-regular maps $f_{i}: N_{i} \rightarrow P(i=0,1)$, we define the sum $\left[f_{0}\right]+\left[f_{1}\right]$ to be the cobordism class of the $\Omega$-regular map $f: N_{0} \cup N_{1} \rightarrow P$ defined by $f \mid N_{i}=f_{i}$. We proceed the arguments commonly in the unoriented case and the 
oriented case in Sections 3 and 4, because in the oriented case we only need to provide manifolds and vector bundles concerned with the orientations. The orientability of $P$ is not necessary even in the oriented case in Theorems 1.1 and Theorem 1.2. However, we will need the orientability of $P$ in the oriented case in Section 8.

We will identify $\mathbb{R}^{m}$ with $S^{m} \backslash\{(0, \cdots, 0,1)\}$ for positive integers $m$ in the following. Let $M$ be an $m$-dimensional compact manifold such that $M$ should be oriented in the oriented case. Let $\ell \gg m$. Take an embedding $e_{M}: M \rightarrow \mathbb{R}^{\ell+m}$ and identify $M$ with $e_{M}(M)$. Let $c_{M}: M \rightarrow G_{m}$ be the classifying map defined by sending a point $x \in M$ to the $m$-plane $T_{x} M \in G_{m}$. Let $v_{M}$ be the orthogonal normal bundle of $M$ in $\mathbb{R}^{\ell+m}$. Let $c_{T M}: T M \rightarrow \gamma_{G_{m}}^{m}$ (respectively $c_{\nu_{M}}: v_{M} \rightarrow \hat{\gamma}_{G_{m}}^{\ell}$ ) be the bundle map covering the classifying map $c_{M}: M \rightarrow G_{m}$, which is defined by sending a vector $\mathbf{v}$ of $T_{x} M$ (respectively $\left.\mathbf{w} \in v_{M, x}\right)$ to $\left(T_{x} M, \mathbf{v}\right)$ (respectively $\left.\left(T_{x} M, \mathbf{w}\right)\right)$. Then we have the canonical trivializations $t_{\boldsymbol{M}}: T M \oplus v_{\boldsymbol{M}} \rightarrow \varepsilon_{\boldsymbol{M}}^{\ell+m}$ and $t_{\boldsymbol{G}_{m}}: \gamma_{\boldsymbol{G}_{m}}^{m} \oplus \hat{\gamma}_{\boldsymbol{G}_{m}}^{\ell} \rightarrow \varepsilon_{\boldsymbol{G}_{m}}^{\ell+m}$. They induce

$$
t_{G_{m}} \circ\left(c_{T M} \oplus c_{v_{M}}\right) \circ t_{M}^{-1}=c_{M} \times \mathrm{id}_{\mathbb{R}^{\ell+m}} .
$$

Let $\mathcal{F}$ be any vector bundle of dimension $q$ over $P$ and $\Omega(m, q)$ be an $L^{k}(q) \times$ $L^{k}(m)$-invariant open subspace of $J^{k}(m, q)$. Let $\boldsymbol{\Omega}_{m}=\Omega\left(\gamma_{G_{m}}^{m}, \mathcal{F}\right)$ be the subbundle of $J^{k}\left(\gamma_{\boldsymbol{G}_{m}}^{m}, \mathcal{F}\right)$ associated to $\Omega(m, q)$. If there is a map $s_{M}: M \rightarrow \boldsymbol{\Omega}_{m}$ with $\pi_{\boldsymbol{G}_{m}}^{k} \circ s_{M}$ being homotopic (respectively equal) to $c_{M}$, then $c_{T M}, c_{\nu_{M}}$ and the projection $\pi_{\boldsymbol{G}_{m}}^{k} \mid \boldsymbol{\Omega}_{m}: \boldsymbol{\Omega}_{m} \rightarrow G_{m}$ induce the bundle maps

$$
c_{s_{M}}^{T M}:\left.T M \rightarrow\left(\pi_{\boldsymbol{G}_{m}}^{k}\right)^{*} \gamma_{\boldsymbol{G}_{m}}^{m}\right|_{\boldsymbol{\Omega}_{m}} \quad \text { and } \quad c_{\boldsymbol{s}_{M}}^{\nu_{M}}: v_{M} \rightarrow\left(\pi_{\boldsymbol{G}_{m}}^{k}\right)^{*} \hat{\gamma}_{\boldsymbol{G}_{m}}^{\ell} \mid \boldsymbol{\Omega}_{m}
$$

covering $s_{M}$ such that $t_{\boldsymbol{\Omega}_{m}} \circ\left(c_{s_{M}}^{T M} \oplus c_{S_{M}}^{v_{M}}\right) \circ t_{M}^{-1}$ is homotopic (respectively equal) to $s_{M} \times \operatorname{id}_{\mathbb{R}^{\ell+m}}$, where

$$
t_{\boldsymbol{\Omega}_{m}}:\left.\left(\pi_{\boldsymbol{G}_{m}}^{k}\right)^{*}\left(\gamma_{\boldsymbol{G}_{m}}^{m} \oplus \hat{\gamma}_{\boldsymbol{G}_{m}}^{\ell}\right)\right|_{\boldsymbol{\Omega}_{m}} \rightarrow \varepsilon_{\boldsymbol{\Omega}_{m}}^{\ell+m}
$$

is the trivialization induced from $t_{G_{m}}$.

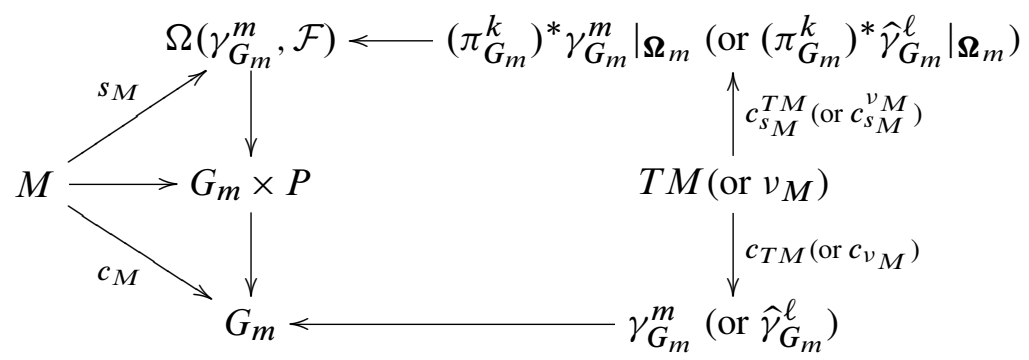

Let $A_{m, q}$ refer to the Grassmann manifold $G_{m, q}$ (respectively oriented Grassmann manifold $\widetilde{G}_{m, q}$ ) of all $m$-subspaces (respectively oriented $m$-subspaces) of $\mathbb{R}^{m+q}$. 
Let $\gamma_{A_{m, q}}^{m}$ and $\widehat{\gamma}_{A_{m, q}}^{q}$ denote the canonical vector bundles of dimensions $m$ and $q$ over $A_{m, q}$ respectively such that

$$
\gamma_{A_{m, q}}^{m} \oplus \hat{\gamma}_{A_{m, q}}^{q}
$$

is the trivial bundle $\varepsilon_{A_{m, q}}^{m+q}$. Let $T\left(\widehat{\gamma}_{A_{m, q}}^{q}\right)$ denote the Thom space of $\hat{\gamma}_{A_{m, q}}^{q}$. Here, we see that the spaces

$$
\left\{T\left(\left(\pi_{\boldsymbol{G}_{m}}^{k}\right)^{*} \hat{\gamma}_{\boldsymbol{G}_{m}}^{\ell} \mid \boldsymbol{\Omega}_{m}\right)\right\}_{\ell}
$$

constitute a spectrum. Let $j: G_{m} \rightarrow A_{m, \ell+1}$ denote the injection mapping an $m$-plane $a$ in $\mathbb{R}^{m+\ell}=\mathbb{R}^{m+\ell} \times 0$ to the same $a$ as the $m$-plane in $\mathbb{R}^{m+\ell+1}$. We have the canonical bundle maps

$$
b: \gamma_{\boldsymbol{G}_{m}}^{m} \longrightarrow \gamma_{A_{m, \ell+1}}^{m} \quad \text { and } \quad \hat{b}: \hat{\gamma}_{\boldsymbol{G}_{m}}^{\ell} \oplus \varepsilon_{\boldsymbol{G}_{m}}^{1} \longrightarrow \hat{\gamma}_{A_{m, \ell+1}}^{\ell+1}
$$

covering $j$. Let $\boldsymbol{\Omega}_{m}^{\bullet}=\Omega\left(\gamma_{A_{m, \ell+1}}^{m}, \mathcal{F}\right)$ be the open subbundle of $J^{k}\left(\gamma_{A_{m, \ell+1}}^{m}, \mathcal{F}\right)$ associated to $\Omega(m, q)$. We have the bundle map

$$
\mathbf{j}\left(b, \operatorname{id}_{\mathcal{F}}\right)_{\Omega}: \boldsymbol{\Omega}_{m} \longrightarrow \boldsymbol{\Omega}_{m}^{\bullet}
$$

covering $j$. Then $\hat{b}$ induces the bundle map

$$
\text { b: }\left.\left.\left(\pi_{\boldsymbol{G}_{m}}^{k}\right)^{*}\left(\hat{\gamma}_{\boldsymbol{G}_{m}}^{\ell} \oplus \varepsilon_{\boldsymbol{G}_{m}}^{1}\right)\right|_{\boldsymbol{\Omega}_{m}} \longrightarrow\left(\pi_{A_{m, \ell+1}}^{k}\right)^{*}\left(\hat{\gamma}_{A_{m, \ell+1}}^{\ell+1}\right)\right|_{\boldsymbol{\Omega}_{m}^{\bullet}}
$$

covering $\mathbf{j}\left(b, \mathrm{id}_{\mathcal{F}}\right)_{\Omega}$. Since

$$
T\left(\left.\left(\pi_{\boldsymbol{G}_{m}}^{k}\right)^{*}\left(\hat{\gamma}_{\boldsymbol{G}_{m}}^{\ell} \oplus \varepsilon_{\boldsymbol{G}_{m}}^{1}\right)\right|_{\boldsymbol{\Omega}_{m}}\right)=T\left(\left.\left(\pi_{\boldsymbol{G}_{m}}^{k}\right)^{*}\left(\hat{\gamma}_{\boldsymbol{G}_{m}}^{\ell}\right)\right|_{\boldsymbol{\Omega}_{m}}\right) \wedge S^{1},
$$

we have the associated map

$$
T(\mathbf{b}): T\left(\left.\left(\pi_{\boldsymbol{G}_{m}}^{k}\right)^{*}\left(\hat{\gamma}_{\boldsymbol{G}_{m}}^{\ell}\right)\right|_{\mathbf{\Omega}_{m}}\right) \wedge S^{1} \longrightarrow T\left(\left.\left(\pi_{A_{m, \ell+1}}^{k}\right)^{*}\left(\hat{\gamma}_{A_{m, \ell+1}}^{\ell+1}\right)\right|_{\mathbf{\Omega}_{m}^{\bullet}}\right) .
$$

This shows the assertion.

Take an embedding $e_{N}: N \rightarrow \mathbb{R}^{n+\ell} \subset S^{n+\ell}$ and apply the above notation. Then we have the bundle map

$$
\mathbf{j}\left(c_{T N}, \operatorname{id}_{T P}\right)_{\Omega}: \Omega(T N, T P) \rightarrow \boldsymbol{\Omega}=\Omega\left(\gamma_{G_{n}}^{n}, T P\right) .
$$

Let $f: N \rightarrow P$ be an $\Omega$-regular map with the jet extension $j^{k} f: N \rightarrow \Omega(T N, T P)$ and let $s_{N}=\mathbf{j}\left(c_{T N}, \operatorname{id}_{T P}\right)_{\Omega} \circ j^{k} f$. Then we have the composite

and

$$
\begin{gathered}
\Delta^{\left(\Omega, \Omega_{\star}\right)} \circ s_{N}: N \rightarrow \Omega_{\star}=\Omega_{\star}\left(\gamma_{G_{n+1}}^{n+1}, T P \oplus \varepsilon_{P}^{1}\right) \\
\mathbf{b}(\hat{\gamma})^{\left(\Omega, \Omega_{\star}\right)} \circ c_{S_{N}}^{\nu_{N}}: v_{N} \rightarrow \hat{\gamma}_{\mathbf{\Omega}_{\star}}^{\ell}
\end{gathered}
$$

covering $\Delta^{\left(\Omega, \Omega_{\star}\right)} \circ s_{N}$. Denote the Pontrjagin-Thom construction (see Thom [57]) for $e_{N}$ by $a_{N}: S^{n+\ell} \rightarrow T\left(v_{N}\right)$. Let $\mathfrak{C}\left(n, P ; \Omega, \Omega_{\star}\right)$ refer to

$$
\mathfrak{N}\left(n, P ; \Omega, \Omega_{\star}\right) \quad \text { or } \quad \mathfrak{O}\left(n, P ; \Omega, \Omega_{\star}\right),
$$


let $\operatorname{Im}\left(T\left(\mathbf{b}(\hat{\gamma})^{\left(\Omega, \Omega_{\star}\right)}\right)\right)$ refer to $\operatorname{Im}^{\mathfrak{N}}\left(T\left(\mathbf{b}(\hat{\gamma})^{\left(\Omega, \Omega_{\star}\right)}\right)\right)$ or $\operatorname{Im}^{\mathcal{O}}\left(T\left(\mathbf{b}(\widehat{\gamma})^{\left(\Omega, \Omega_{\star}\right)}\right)\right)$ and let $\omega$ refer to $\mathfrak{n}^{\left(\Omega, \Omega_{\star}\right)}$ or $\mathfrak{o}^{\left(\Omega, \Omega_{\star}\right)}$, depending on whether we work in the unoriented case or oriented case. We now define the maps

$$
\omega: \mathfrak{C}\left(n, P ; \Omega, \Omega_{\star}\right) \longrightarrow \operatorname{Im}\left(T\left(\mathbf{b}(\widehat{\gamma})^{\left(\Omega, \Omega_{\star}\right)}\right)\right)
$$

by mapping the cobordism class $[f]$ of $\mathfrak{C}\left(n, P ; \Omega, \Omega_{\star}\right)$ to the homotopy class of $T\left(\mathbf{b}(\widehat{\gamma})^{\left(\Omega, \Omega_{\star}\right)}\right) \circ T\left(c_{S_{N}}^{\nu_{N}}\right) \circ a_{N}$.

We have to prove that $\omega([f])$ does not depend on the choice of a representative $f$.

Lemma 3.1 Suppose that two $\Omega$-regular maps $f_{i}: N_{i} \rightarrow P(i=0,1)$ are $\Omega_{\star}-$ cobordant. Then we have $\mathfrak{n}^{\left(\Omega, \Omega_{\star}\right)}\left(\left[f_{0}\right]\right)=\mathfrak{n}^{\left(\Omega, \Omega_{\star}\right)}\left(\left[f_{1}\right]\right)$. If $N_{i}$ are oriented and $f_{i}$ $(i=0,1)$ are oriented $\Omega_{\star}$-cobordant, then we have $\mathfrak{o}^{\left(\Omega, \Omega_{\star}\right)}\left(\left[f_{0}\right]\right)=\mathfrak{o}^{\left(\Omega, \Omega_{\star}\right)}\left(\left[f_{1}\right]\right)$.

Proof We first have to prove that $\omega([f])$ does not depend on the choice of an embedding $e_{N}$. Let $\ell$ be a sufficiently large integer. Let $e_{N}: N \rightarrow \mathbb{R}^{n+\ell}$ be an embedding of $N$. For a nonnegative integer $m$, let $e_{N}^{m}$ be the composite of $e_{N}$ and the inclusion $\mathbb{R}^{n+\ell}=\mathbb{R}^{n+\ell} \times 0 \rightarrow \mathbb{R}^{n+\ell+m}$. Then we may regard $v_{N} \oplus \varepsilon_{N}^{m}$ as the normal bundle of $e_{N}^{m}$ and the Pontrjagin-Thom construction for $e_{N}^{m}$ is the $m$-th suspension $S^{n+\ell+m} \rightarrow T\left(v_{N} \oplus \varepsilon_{N}^{m}\right)=T\left(v_{N}\right) \wedge S^{m}$ of that for $e_{N}$.

Given two embeddings $e_{N_{i}}: N_{i} \rightarrow \mathbb{R}^{n+\ell_{i}}(i=0,1)$ with $\ell_{1}=\ell_{0}+m$ for $m \geqq 0$, by the above argument we consider $e_{N_{0}}^{m}$ in place of $e_{N_{0}}$. It will be proved by the argument below that $\omega([f])$ does not depend on the choice of $e_{N_{0}}^{m}$ and $e_{N_{1}}$.

Let $\epsilon$ be a sufficiently small positive real number. Let $I(0, \epsilon)$ and $I(1, \epsilon)$ denote the intervals $[0, \epsilon]$ and $[1-\epsilon, 1]$ respectively. Let $\mathfrak{C}:(W, \partial W) \rightarrow(P \times[0,1], P \times 0 \cup P \times 1)$ be an $\Omega_{\star}$-cobordism of $f_{0}$ and $f_{1}$. Take embeddings $e_{N_{i}}: N_{i} \rightarrow \mathbb{R}^{n+\ell}$ and $e_{W}: W \rightarrow$ $\mathbb{R}^{n+\ell} \times[0,1]$, and let us identify as $N_{i}=e_{N_{i}}\left(N_{i}\right)=e_{N_{i}}\left(N_{i}\right) \times\{i\}, W=e_{W}(W)$ and $P=P \times\{i\}$. Then we may assume that for $i=0,1$,

(i) $W \cap\left(S^{n+\ell} \times I(i, \epsilon)\right)=N_{i} \times I(i, \epsilon)$,

(ii) $e_{W} \mid N_{i} \times I(i, \epsilon)=e_{N_{i}} \times \operatorname{id}_{I(i, \epsilon)}$,

(iii) $\mathfrak{C} \mid N_{i} \times I(i, \epsilon)=f_{i} \times \mathrm{id}_{I(i, \epsilon)}$,

(iv) $j^{k} \mathfrak{C}\left|N_{i} \times\{i\}=j^{k}\left(f_{i} \times \operatorname{id}_{\mathbb{R}}\right)\right| N_{i} \times\{i\}$ under $N_{i}=N_{i} \times\{i\}$ and $P=P \times\{i\}$.

In the identification $\left.T W\right|_{N_{i}}=T N_{i} \oplus \varepsilon_{N_{i}}^{1}$, the positive direction of $\varepsilon_{N_{0}}^{1}$ should correspond to the inward normal direction, and that of $\varepsilon_{N_{1}}^{1}$ should correspond to the outward normal direction. Then we may assume that the trivializations

$$
t_{N_{i}}: T N_{i} \oplus v_{N_{i}} \rightarrow \varepsilon_{N_{i}}^{n+\ell} \text { and } t_{W}: T W \oplus v_{W} \rightarrow \varepsilon_{W}^{n+\ell+1}
$$


satisfy $\left.t_{W}\right|_{N_{i}}=\left(t_{N_{i}} \oplus \operatorname{id}_{\varepsilon_{N_{i}}^{1}}^{1}\right) \circ\left(\operatorname{id}_{T N_{i}} \oplus \mathbf{k}_{N_{i}}^{\sim}\right)$, where $\mathbf{k}_{N_{i}}^{\sim}: \varepsilon_{N_{i}}^{1} \oplus v_{N_{i}} \rightarrow v_{N_{i}} \oplus \varepsilon_{N_{i}}^{1}$ is the map interchanging the components $\varepsilon_{N_{i}}^{1}$ and $v_{N_{i}}$. Let

$$
c_{\gamma_{n}}^{n} \oplus \varepsilon_{G_{n}}^{1}: \gamma_{G_{n}}^{n} \oplus \varepsilon_{G_{n}}^{1} \rightarrow \gamma_{G_{n+1}}^{n+1}
$$

denote the bundle map covering $i^{G}: G_{n} \rightarrow G_{n+1}$ defined by

$$
c_{\gamma_{G_{n}}^{n} \oplus \varepsilon_{G_{n}}^{1}}^{1}\left(V_{x} \oplus(x, t)\right)=V_{i}(x)+t \mathbf{e}_{n+\ell+1}
$$

for $x \in G_{n}, V_{x} \in\left(\gamma_{G_{n}}^{n}\right)_{x}$ and $t \in \mathbb{R}$. Let $s_{N_{i}}=\mathbf{j}\left(c_{T N_{i}}, \operatorname{id}_{T P}\right)_{\Omega} \circ j^{k} f_{i}$ and $s_{W}=$ $\mathbf{j}\left(c_{T W}, \operatorname{id}_{T(P \times[0,1])}\right)_{\Omega_{\star}} \circ j^{k} \mathfrak{C}$. Then we have that

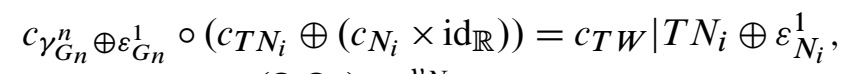

$$
\begin{aligned}
& \left.c_{S_{W}}^{\nu_{W}}\right|_{N_{i}}=\mathbf{b}(\widehat{\gamma})^{\left(\Omega, \Omega_{\star}\right)} \circ c_{S_{N_{i}}}^{\nu_{N_{i}}}
\end{aligned}
$$

Let $a_{W}: S^{n+\ell} \times[0,1] \rightarrow T\left(v_{W}\right)$ be the Pontrjagin-Thom construction for $e_{W}$. Under the identifications

$$
\begin{gathered}
\Omega_{\star}(T W, T(P \times[0,1]))=\Omega_{\star}\left(T W, T P \oplus \varepsilon_{P}^{1}\right) \times[0,1], \\
\Omega_{\star}\left(\gamma_{G_{n+1}}^{n+1}, T(P \times[0,1])\right)=\Omega_{\star}\left(\gamma_{G_{n+1}}^{n+1}, T P \oplus \varepsilon_{P}^{1}\right) \times[0,1],
\end{gathered}
$$

the composite $T\left(c_{s_{W}}^{v_{W}}\right) \circ a_{W}$ gives a homotopy between $T\left(\mathbf{b}(\widehat{\gamma})^{\left(\Omega, \Omega_{\star}\right)}\right) \circ T\left(c_{s_{N_{0}}}^{\nu_{N_{0}}}\right) \circ a_{N_{0}}$ and $T\left(\mathbf{b}(\hat{\gamma})^{\left(\Omega, \Omega_{\star}\right)}\right) \circ T\left(c_{S_{N_{1}}}\right) \circ a_{N_{1}}$. This proves $\omega\left(\left[f_{0}\right]\right)=\omega\left(\left[f_{1}\right]\right)$.

\section{Proof of Theorem 1.1}

We prove Theorem 1.1 in this section. Let $\Omega(n, p)$ and $\Omega_{\star}(n+1, p+1)$ be the open subsets given in Theorem 1.1.

Proof of Theorem 1.1 We use the notation in the proof of Lemma 3.1. We first prove that $\omega$ is injective. For this, take two $\Omega$-regular maps $f_{i}: N_{i} \rightarrow P(i=0,1)$ such that $\omega\left(\left[f_{0}\right]\right)=\omega\left(\left[f_{1}\right]\right)$. Recall the map $T\left(\mathbf{b}(\widehat{\gamma})^{\left(\Omega, \Omega_{\star}\right.}\right) \circ T\left(c_{S_{N_{i}}}^{\nu_{N_{i}}}\right) \circ a_{N_{i}}$ which represents $\omega\left(\left[f_{i}\right]\right)$. There is a homotopy $H: S^{n+\ell} \times[0,1] \rightarrow T\left(\hat{\gamma}_{\mathbf{\Omega}_{\star}}^{\ell}\right)$ such that if $\epsilon$ is sufficiently small, then we have, for $i=0,1$,

(i) $H \mid S^{n+\ell} \times I(i, \epsilon)=T\left(\mathbf{b}(\widehat{\gamma})^{\left(\Omega, \Omega_{\star}\right)}\right) \circ T\left(c_{S_{N_{i}}}^{v_{N_{i}}}\right) \circ a_{N_{i}} \circ\left(\pi_{S^{n+\ell}} \mid S^{n+\ell} \times I(i, \epsilon)\right)$,

(ii) $H$ is smooth around $H^{-1}\left(\boldsymbol{\Omega}_{\star}\right)$ and is transverse to $\boldsymbol{\Omega}_{\star}$.

We set $W=H^{-1}\left(\boldsymbol{\Omega}_{\star}\right)$. Then we have

(iii) $W \cap\left(S^{n+\ell} \times I(i, \epsilon)\right)=N_{i} \times I(i, \epsilon)$,

(iv) $H \mid N_{i} \times I(i, \epsilon)=\Delta^{\left(\Omega, \Omega_{\star}\right)} \circ s_{N_{i}} \circ\left(\pi_{N_{i}} \mid\left(N_{i} \times I(i, \epsilon)\right)\right.$, 
(v) $\left.T W\right|_{N_{i} \times I(i, \epsilon)}=T\left(N_{i} \times I(i, \epsilon)\right)=\left(T N_{i} \oplus \varepsilon_{N_{i}}^{1}\right) \times I(i, \epsilon)$,

(vi) $\left.v_{W}\right|_{N_{i} \times I(i, \epsilon)}=v_{N_{i}} \times I(i, \epsilon)$.

By (ii) we have the bundle map $c_{\sim}^{\nu_{W}}: v_{W} \rightarrow \hat{\gamma}_{\mathbf{\Omega}_{\star}}^{\ell}$ covering $H \mid W: W \rightarrow \boldsymbol{\Omega}_{\star}$ such that (vii) $\left.\quad c_{\sim}^{\nu_{W}}\right|_{N_{i} \times I(i, \epsilon)}=\mathbf{b}(\widehat{\gamma})^{\left(\Omega, \Omega_{\star}\right)} \circ c_{S_{N_{i}}}^{\nu_{N_{i}}} \circ\left(\pi_{v_{N_{i}}} \mid v_{N_{i}} \times I(i, \epsilon)\right)$ by (i) and (iv).

By [4, Proposition 3.3] we obtain a bundle map of $T W \oplus \varepsilon_{W}^{3}$ to

$$
\left(\pi_{G_{n+1}}^{k}\right)^{*}\left(\gamma_{G_{n+1}}^{n+1} \oplus \varepsilon_{G_{n+1}}^{3}\right) \mid \mathbf{\Omega}_{\star}
$$

with a required property concerning the trivialization. By this property and the dimensional reason, we obtain a bundle map

$$
c_{\sim}^{T W}:\left.T W \rightarrow\left(\pi_{G_{n+1}}^{k}\right)^{*}\left(\gamma_{G_{n+1}}^{n+1}\right)\right|_{\mathbf{\Omega}_{\star}}
$$

covering $H \mid W: W \rightarrow \boldsymbol{\Omega}_{\star}$ induced from the above bundle map such that $t_{\mathbf{\Omega}_{\star}} \circ\left(c_{\sim}^{T} W^{W} \oplus\right.$ $\left.c_{\sim}^{v_{W}}\right) \circ t_{W}^{-1}$ is homotopic to $(H \mid W) \times \operatorname{id}_{\mathbb{R}^{n+\ell+1}}$. Since $\gamma_{G_{n+1}}^{n+1}$ is the universal bundle $(\ell \gg n), c_{\sim}^{T W}$ is regarded as $c_{H \mid W}^{T W}$. Let

$$
\mathbf{b}\left(\gamma \oplus \varepsilon^{1}\right)^{\left(\Omega, \Omega_{\star}\right)}:\left.\left.\left(\pi_{\boldsymbol{G}_{n}}^{k}\right)^{*}\left(\gamma_{\boldsymbol{G}_{n}}^{n} \oplus \varepsilon_{\boldsymbol{G}_{n}}^{1}\right)\right|_{\boldsymbol{\Omega}} \rightarrow\left(\pi_{\boldsymbol{G}_{n+1}}^{k}\right)^{*}\left(\gamma_{\boldsymbol{G}_{n+1}}^{n+1}\right)\right|_{\boldsymbol{\Omega}_{\star}}
$$

covering $\Delta^{\left(\Omega, \Omega_{\star}\right)}$ be the bundle map induced from $c_{\gamma_{G_{n}}^{n} \oplus \varepsilon_{G_{n}}^{1}}$ in the proof of Lemma 3.1. Then we may assume by (iv), (v) and (vi) that

(viii) $\left.c_{\sim}^{T W}\right|_{\left(N_{i} \times I(i, \epsilon)\right)}$ is equal to

$$
\mathbf{b}\left(\gamma \oplus \varepsilon^{1}\right)^{\left(\Omega, \Omega_{\star}\right)} \circ\left(c_{s_{N_{i}}}^{T N_{i}} \oplus\left(c_{S_{N_{i}}} \times \mathrm{id}_{\mathbb{R}}\right)\right) \circ \pi_{T N_{i} \oplus \varepsilon_{N_{i}}} \mid\left(\left(T N_{i} \oplus \varepsilon_{N_{i}}\right) \times I(i, \epsilon)\right) .
$$

Hence, $c_{W}$ is homotopic to $\pi_{G_{n+1}}^{k} \circ H \mid W$ relative to $\left(N_{0} \times[0, \epsilon]\right) \cup\left(N_{1} \times[1-\epsilon, 1]\right)$. Let $\pi_{T P \oplus \varepsilon_{P}^{1}}: T(P \times[0,1])=\left(T P \oplus \varepsilon_{P}^{1}\right) \times[0,1] \rightarrow T P \oplus \varepsilon_{P}^{1}$ be the canonical bundle map covering the canonical projection $\pi_{P}: P \times[0,1] \rightarrow P$. Then we have the bundle map

$\mathbf{j}\left(c_{T W}, \pi_{T P \oplus \varepsilon_{P}^{1}}\right)_{\Omega_{\star}}: \Omega_{\star}(T W, T(P \times[0,1]))=\Omega_{\star}\left(T W, T P \oplus \varepsilon_{P}^{1}\right) \times[0,1]$

$$
\longrightarrow \Omega_{\star}=\Omega_{\star}\left(\gamma_{G_{n+1}^{n+1}}^{n+1}, T P \oplus \varepsilon_{P}^{1}\right)
$$

covering $c_{W} \times \pi_{P}$. Therefore, since $[0,1]$ is contractible, there is a section $s_{W}: W \rightarrow$ $\Omega_{\star}(T W, T(P \times[0,1]))$ such that

$$
\begin{aligned}
\pi_{P \times[0,1]}^{k} \circ s_{W} \mid N_{i} \times I(i, \epsilon) & =f_{i} \times \operatorname{id}_{I(i, \epsilon)}, \\
\pi_{P} \circ \pi_{P \times[0,1]}^{k} \circ s_{W} & =\pi_{P}^{k} \circ(H \mid W),
\end{aligned}
$$


and that $\mathbf{j}\left(c_{T W}, \pi_{T P \oplus \varepsilon_{P}^{1}}\right)_{\Omega_{\star}} \circ s_{W}$ is homotopic to $H \mid W$ relative to $\left(N_{0} \times[0, \epsilon]\right) \cup$ $\left(N_{1} \times[1-\epsilon, 1]\right)$.

Since $\Omega_{\star}(T W, T(P \times[0,1]))$ satisfies the relative h-principle on the existence level, there exists an $\Omega_{\star}$-regular map $\mathfrak{C}: W \rightarrow P \times[0,1]$ such that $\mathfrak{C}(x, t)=f_{0}(x) \times t$ for $0 \leqq t \leqq \epsilon, \mathfrak{C}(x, t)=f_{1}(x) \times t$ for $1-\epsilon \leqq t \leqq 1$ and that $j^{k} \mathfrak{C}$ is homotopic to $s_{W}$ relative to $\left(N_{0} \times[0, \epsilon / 2]\right) \cup\left(N_{1} \times[1-\epsilon / 2,1]\right)$. This implies that the $\Omega$-regular maps $f_{0}$ and $f_{1}$ are $\Omega_{\star}-$ cobordant. This proves that $\omega$ is injective.

We next prove that $\omega$ is surjective. Let an element $\tilde{\alpha}$ of $\operatorname{Im}\left(T\left(\mathbf{b}(\hat{\gamma})^{\left(\Omega, \Omega_{\star}\right)}\right)\right)$ be represented by a map $\alpha: S^{n+\ell} \rightarrow T\left(\hat{\gamma}_{\mathbf{\Omega}}^{\ell}\right)$ such that $\left(T\left(\mathbf{b}(\hat{\gamma})^{\left(\Omega, \Omega_{\star}\right)}\right)\right)_{*}([\alpha])=\widetilde{\alpha}$. We may suppose that $\alpha$ is smooth around $\alpha^{-1}(\boldsymbol{\Omega})$ and is transverse to $\Omega$. We set $N=$ $\alpha^{-1}(\boldsymbol{\Omega})$. If $N=\varnothing$, then $[\alpha]$ must be a null element, although we can deform $\alpha$ so that $N \neq \varnothing$ even in this case. Since $\alpha$ is transverse to $\boldsymbol{\Omega}$, we have the bundle map $c_{\sim}^{\nu_{N}}: v_{N} \rightarrow \hat{\gamma}_{\mathbf{\Omega}}^{\ell}$ covering $\alpha \mid N$. It follows from [4, Proposition 3.3] that there exists a bundle map

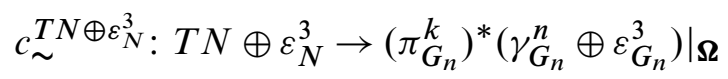

covering $\alpha \mid N: N \rightarrow \boldsymbol{\Omega}$ such that the composite

$$
\begin{aligned}
& \left(t_{\mathbf{\Omega}} \oplus \operatorname{id}_{\varepsilon_{\boldsymbol{\Omega}}^{3}}^{3}\right) \circ\left(\operatorname{id}_{\left(\pi_{G_{n}}^{k}\right) *\left(\gamma_{G_{n}}^{n}\right)} \oplus \mathbf{k}_{G_{n}}^{\tilde{H}_{n}}\right)
\end{aligned}
$$

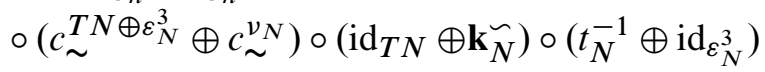

is homotopic to $(\alpha \mid N) \times \mathrm{id}_{\mathbb{R}^{n+\ell+3}}$, where $\mathbf{k}_{G_{n}}^{\varkappa}: \varepsilon_{G_{n}}^{3} \oplus \hat{\gamma}_{G_{n}}^{\ell} \rightarrow \hat{\gamma}_{G_{n}}^{\ell} \oplus \varepsilon_{G_{n}}^{3}$ and $\mathbf{k}_{N}^{\varkappa}: v_{N} \oplus$ $\varepsilon_{N}^{3} \rightarrow \varepsilon_{N}^{3} \oplus v_{N}$ are the maps interchanging the components respectively. Since $\gamma_{G_{n}}^{n}$ is

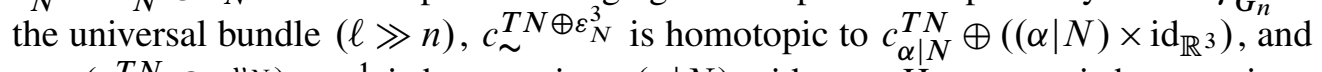
$t_{\mathbf{\Omega}} \circ\left(c_{\alpha \mid N}^{T N} \oplus c_{\sim}^{\nu_{N}}\right) \circ t_{N}^{-1}$ is homotopic to $(\alpha \mid N) \times \mathrm{id}_{\mathbb{R}^{n+\ell}}$. Hence, $c_{N}$ is homotopic to $\pi_{G_{n}}^{k} \circ \alpha \mid N$. By [4, Proposition 3.3] again, $c_{\sim}^{\nu_{N}}$ and $c_{\alpha \mid N}^{\nu_{N}}$ are homotopic as bundle maps $v_{N} \rightarrow \hat{\gamma}_{\mathbf{\Omega}}^{\ell}$. Since we have the bundle map

$$
\mathbf{j}\left(c_{T N}, \operatorname{id}_{T P}\right)_{\boldsymbol{\Omega}}: \Omega(T N, T P) \longrightarrow \boldsymbol{\Omega}=\Omega\left(\gamma_{G_{n}}^{n}, T P\right)
$$

covering $c_{N} \times \operatorname{id}_{P}$, there is a section $s_{N}: N \rightarrow \Omega(T N, T P)$ such that $\pi_{P}^{k} \circ s_{N}=$ $\pi_{P}^{k} \circ \alpha \mid N$ and $\mathbf{j}\left(c_{T N}, \operatorname{id}_{T P}\right)_{\boldsymbol{\Omega}} \circ s_{N}$ is homotopic to $\alpha \mid N$. Since $\Omega(T N, T P)$ satisfies the h-principle, there exists an $\Omega$-regular map $f: N \rightarrow P$ such that $j^{k} f$ is homotopic to $s_{N}$. This implies that $\mathbf{j}\left(c_{T N}, \operatorname{id}_{T P}\right)_{\boldsymbol{\Omega}} \circ j^{k} f$ and $\alpha \mid N$ are homotopic. This proves that

$$
\begin{aligned}
\omega([f]) & =\left[T\left(\mathbf{b}(\hat{\gamma})^{\left(\Omega, \Omega_{\star}\right)}\right) \circ T\left(c_{\alpha \mid N}^{\nu_{N}}\right) \circ a_{N}\right] \\
& =\left[T\left(\mathbf{b}(\hat{\gamma})^{\left(\Omega, \Omega_{\star}\right)}\right) \circ T\left(c_{\sim}^{\nu_{N}}\right) \circ a_{N}\right] \\
& =\left[T\left(\mathbf{b}(\hat{\gamma})^{\left(\Omega, \Omega_{\star}\right)}\right) \circ \alpha\right] \\
& =\tilde{\alpha} .
\end{aligned}
$$


This is what we want.

Under the assumption of Theorem $1.1 \mathfrak{C}\left(n, P ; \Omega, \Omega_{\star}\right)$ inherits the structure of an abelian group from the stable homotopy groups. The null element is defined to be represented by an $\Omega$-regular map $f: N \rightarrow P$, which has an $\Omega_{\star}$-cobordism

$$
\mathfrak{C}:(W, \partial W) \rightarrow(P \times[0,1], P \times 0)
$$

with $\partial W=N$ such that $\mathfrak{C} \mid N=f$ under the identification $P \times 0=P$.

\section{Examples of $\mathcal{K}$-invariant open subsets}

In this section $n<p$ is not necessarily assumed. As an important example of $\Omega_{*}(n+$ $1, p+1)$ for $\Omega(n, p)$ we recall $\Omega_{\mathcal{K}}^{1}=\Omega_{\mathcal{K}}(n+1, p+1)$, which is the set consisting of all $\mathcal{K}$-orbits $\mathcal{K}\left(i_{+1}(z)\right)$ for $k$-jets $z \in \Omega(n, p)$. Let $\mathcal{C}_{m}$ denote the ring of smooth function germs $\left(\mathbb{R}^{m}, 0\right) \rightarrow \mathbb{R}$ and let $\mathfrak{m}_{m}$ denote its maximal ideal.

Lemma 5.1 Let $i$ be a nonnegative integer smaller than $n+1$. Then any $k$-jet $w \in \Sigma^{i}(n+1, p+1)$ has a $k$-jet $z \in \Sigma^{i}(n, p)$ such that $w$ lies in $\mathcal{K}\left(i_{+1}(z)\right)$.

Proof We consider the usual coordinates $x=\left(x_{1}, x_{2}, \cdots, x_{n+1}\right)$ of $\mathbb{R}^{n+1}$ and $y=$ $\left(y_{1}, y_{2}, \cdots, y_{p+1}\right)$ of $\mathbb{R}^{p+1}$. Under suitable respective coordinates

$$
x^{\prime}=\left(x_{1}^{\prime}, x_{2}^{\prime}, \cdots, x_{n+1}^{\prime}\right) \text { and } y^{\prime}=\left(y_{1}^{\prime}, y_{2}^{\prime}, \cdots, y_{p+1}^{\prime}\right)
$$

of $\mathbb{R}^{n+1}$ and $\mathbb{R}^{p+1}, w$ is represented as $w=j_{0}^{k} g$ with

$$
\left(y_{1}^{\prime} \circ g\left(x^{\prime}\right), \cdots, y_{p+1}^{\prime} \circ g\left(x^{\prime}\right)\right)=\left(x_{1}^{\prime}, \cdots, x_{n-i}^{\prime}, g^{n-i+1}\left(x^{\prime}\right), \cdots, g^{p}\left(x^{\prime}\right), x_{n+1}^{\prime}\right) \text {, }
$$

where $g^{j} \in \mathfrak{m}_{n+1}^{2}$. Let $\bar{g}: \mathbb{R}^{n+1} \rightarrow \mathbb{R}^{p+1}$ be the map germ defined by

$$
\left(y_{1} \circ \bar{g}(x), \cdots, y_{p+1} \circ \bar{g}(x)\right)=\left(x_{1}, \cdots, x_{n-i}, g^{n-i+1}(x), \cdots, g^{p}(x), x_{n+1}\right) .
$$

It is evident that $j_{0}^{k} \bar{g} \in \mathcal{K}(w)$. Let $\stackrel{\circ}{x}=\left(x_{1}, \cdots, x_{n}, 0\right)$. Define the map germ $f: \mathbb{R}^{n}=$ $\mathbb{R}^{n} \times 0 \rightarrow \mathbb{R}^{p}$ by

$$
f(\stackrel{\circ}{x})= \begin{cases}\left(x_{1}, \cdots, x_{n-i}, g^{n-i+1}(\stackrel{\circ}{x}), g^{n-i+2}(\stackrel{\circ}{x}), \cdots, g^{p}(\stackrel{\circ}{x})\right) & \text { for } i<n, \\ \left(g^{1}(\stackrel{\circ}{x}), g^{2}(\stackrel{\circ}{x}), \cdots, g^{p}(\stackrel{\circ}{x})\right) & \text { for } i=n .\end{cases}
$$

Then we have

$$
\begin{aligned}
Q\left(j_{0}^{k} \bar{g}\right) & =\mathcal{C}_{n+1} /\left(\bar{g}^{*}\left(\mathfrak{m}_{p+1}\right)+\mathfrak{m}_{n+1}^{k+1}\right) \\
& \approx \mathcal{C}_{n} /\left(f^{*}\left(\mathfrak{m}_{p}\right)+\mathfrak{m}_{n}^{k+1}\right) \\
& =Q\left(j_{0}^{k} f\right) .
\end{aligned}
$$


Setting $z=j_{0}^{k} f$, we have $w \in \mathcal{K}\left(i_{+1}(z)\right)$.

Lemma 5.2 Let $\Omega(n, p)$ be a $\mathcal{K}$-invariant open subset of $J^{k}(n, p)$. Then the set $\Omega_{\mathcal{K}}^{m}$ is open in $J^{k}(n+m, p+m)$ for $m>0$.

Proof It is enough to prove the case $m=1$. Suppose to the contrary that $\Omega_{\mathcal{K}}^{1}$ is not open. Then there exists a $k$-jet $w \in \Omega_{\mathcal{K}}^{1}$ and $k$-jets $w_{j} \notin \Omega_{\mathcal{K}}^{1}$ such that $\lim _{j \rightarrow \infty} w_{j}=$ $w$. By definition, there exists a $k$-jet $z \in \Omega(n, p)$ such that $w \in \mathcal{K}\left(i_{+1}(z)\right)$. Let $i=n-\operatorname{rank} z$. Then we may assume without loss of generality that $w=i_{+1}(z)$, $w=j_{0}^{k} g, w_{j}=j_{0}^{k} g_{j}$ and $\lim _{j \rightarrow \infty} w_{j}=w$ with

$$
\begin{aligned}
\left(y_{1} \circ g(x), \cdots, y_{p+1} \circ g(x)\right) & =\left(x_{1}, \cdots, x_{n-i}, g^{n-i+1}(x), \cdots, g^{p}(x), x_{n+1}\right), \\
\left(y_{1} \circ g_{j}(x), \cdots, y_{p+1} \circ g_{j}(x)\right) & =\left(g_{j}^{1}(x), \cdots, g_{j}^{n-i}(x), g_{j}^{n-i+1}(x), \cdots, g_{j}^{p+1}(x)\right),
\end{aligned}
$$

where $g^{t} \in \mathfrak{m}_{n}^{2}$ for $n-i+1 \leqq t \leqq p$. Since $\lim _{j \rightarrow \infty} w_{j}=i_{+1}(z)$, we set

$$
\begin{aligned}
& h_{j}^{1}\left(x_{1}, \cdots, x_{n+1}\right)=\left(x_{1}, \cdots, x_{n-i}, g_{j}^{n-i+1}(x), \cdots, g_{j}^{p}(x), x_{n+1}\right), \\
& h_{j}^{2}\left(x_{1}, \cdots, x_{n+1}\right)=\left(x_{1}, \cdots, x_{n-i}, g_{j}^{n-i+1}(\stackrel{\circ}{x}), \cdots, g_{j}^{p}(\stackrel{\circ}{x}), x_{n+1}\right) .
\end{aligned}
$$

Since the map germ defined by

$$
\left(x_{1}, \cdots, x_{n+1}\right) \longmapsto\left(g_{j}^{1}(x), \cdots, g_{j}^{n-i}(x), x_{n-i+1}, \cdots, x_{n}, g_{j}^{p+1}(x)\right)
$$

is a local diffeomorphism for sufficiently large numbers $j$, we have

$$
Q_{k}\left(w_{j}\right) \approx Q_{k}\left(j_{0}^{k} h_{j}^{1}\right) \approx Q_{k}\left(j_{0}^{k} h_{j}^{2}\right) .
$$

Let us define

$$
f_{j}\left(x_{1}, \cdots, x_{n}\right)= \begin{cases}\left(x_{1}, \cdots, x_{n-i}, g_{j}^{n-i+1}(\stackrel{\circ}{x}), \cdots, g_{j}^{p}(\stackrel{\circ}{x})\right) & \text { for } i<n, \\ \left(g_{j}^{1}(\stackrel{\circ}{x}), \cdots, g_{j}^{p}(\stackrel{\circ}{x})\right) & \text { for } i=n .\end{cases}
$$

Then we have that $\lim _{j \rightarrow \infty} j_{0}^{k} h_{j}^{1}=w=i_{+1}(z)$ and $i_{+1}\left(j_{0}^{k} f_{j}\right)=j_{0}^{k} h_{j}^{2}$, and hence, $Q_{k}\left(w_{j}\right) \approx Q_{k}\left(j_{0}^{k} f_{j}\right)$. Since $\lim _{j \rightarrow \infty} j_{0}^{k} f_{j}=z$, we have that $j_{0}^{k} f_{j} \in \Omega(n, p)$ for sufficiently large numbers $j$. By definition, we have $j_{0}^{k} h_{j}^{2} \in \Omega_{\mathcal{K}}^{1}$ for sufficiently large numbers $j$. Since $Q_{k}\left(w_{j}\right) \approx Q_{k}\left(j_{0}^{k} h_{j}^{2}\right)$, it follows from [38, Theorem 2.1] that $w_{j} \in \Omega_{\mathcal{K}}^{1}$ for sufficiently large numbers $j$. This is a contradiction.

Lemma 5.3 If two map germs $f_{1}, f_{2}:\left(\mathbb{R}^{m}, 0\right) \rightarrow\left(\mathbb{R}^{q}, 0\right)$ are $\mathcal{K}$-equivalent, then the Boardman symbols of $j_{0}^{k} f_{1}$ and $j_{0}^{k} f_{2}$ are the same. Consequently, the Boardman manifold $\Sigma^{I}(m, q)$ is invariant with respect to the action of $\mathcal{K}$. 
Proof By [36] there exist a germ of a diffeomorphism $h:\left(\mathbb{R}^{m}, 0\right) \rightarrow\left(\mathbb{R}^{m}, 0\right)$ and a smooth map germ $M:\left(\mathbb{R}^{m}, 0\right) \rightarrow G L(q)$ such that $M(x) f_{1}(h(x))=f_{2}(x)$. Let $f_{i}(x)=\left(f_{1}^{i}(x), \cdots, f_{q}^{i}(x)\right)$ with $f_{j}^{i} \in \mathcal{C}_{m} / \mathfrak{m}_{m}^{k+1}(i=1,2)$. Let $\Im\left(f_{i}\right)$ denote the ideal of $\mathcal{C}_{m} / \mathfrak{m}_{m}^{k+1}$ generated by $f_{1}^{i}, \cdots, f_{q}^{i}$. Let $h_{*}: \mathcal{C}_{m} / \mathfrak{m}_{m}^{k+1} \rightarrow \mathcal{C}_{m} / \mathfrak{m}_{m}^{k+1}$ be the isomorphism defined by $h_{*}(\phi)=\phi \circ h$. Then we have $h_{*}\left(\mathfrak{I}\left(f_{1}\right)\right)=\mathfrak{I}\left(f_{2}\right)$. The Boardman symbols of $j_{0}^{k} f_{i}(i=1,2)$ are determined by $\Im\left(f_{i}\right)$ and are the same by [39]. This proves the assertion.

Lemma 5.4 If $I$ is a Boardman symbol such that $\Omega^{I}(n, p)$ is nonempty, then $\Omega^{I}(n+$ $1, p+1)$ is the union of all $\mathcal{K}$-orbits $\mathcal{K}\left(i_{+1}(z)\right)$ for $z \in \Omega^{I}(n, p)$.

Proof Let $I=\left(i_{1}, i_{2}, \cdots\right)$. Since $\Omega^{I}(n, p)$ is nonempty, we have $i_{1} \leqq n$. Let $w \in \Omega^{I}(n+1, p+1)$, whose Boardman symbol is $J \leqq I$. Since $j_{1} \leqq i_{1} \leqq n$, it follows from Lemma 5.1 that there exists a $z \in \Omega^{I}(n, p)$ such that $w \in \mathcal{K}\left(i_{+1}(z)\right)$.

Conversely, let $z \in \Omega^{I}(n, p)$ with Boardman symbol $K \leqq I$. Since the Boardman symbol of $i_{+1}(z)$ is obviously equal to $K$, we have $i_{+1}(z) \in \Omega^{I}(n+1, p+1)$. This shows the assertion.

\section{Preliminaries for Theorem 1.2}

We prepare lemmas and propositions for the proof of Theorem 1.2.

Let $\mathbf{e}_{i}=(0, \cdots, 0,1,0, \cdots, 0)$ with 1 being the $i$-th component. Let $\operatorname{pr}_{p+1}: \mathbb{R}^{p+1} \rightarrow$ $\mathbb{R}$ be the projection mapping $\left(x_{1}, \cdots, x_{p+1}\right)$ to $x_{p+1}$. Let $\pi_{1}^{k}: J^{k}(n, p) \rightarrow J^{1}(n, p)$ be the canonical forgetting projection.

Let $K$ be a finite simplicial complex and $L$ be its subcomplex such that $K \backslash L$ is a manifold and $\operatorname{dim} L<\operatorname{dim} K$.

Lemma 6.1 Let $\Omega(n, p)$ be a $\mathcal{K}$-invariant open subset of $J^{k}(n, p)$. Let $(K, L)$ be given as above and $\operatorname{dim} K<p$. Let $\psi:(K, L) \rightarrow\left(\Omega_{\mathcal{K}}^{1}, i_{+1}(\Omega(n, p))\right)$ be a map such that $\psi \mid(K \backslash L)$ is smooth. Then there exists a homotopy $\psi_{\lambda}:(K, L) \rightarrow$ $\left(\Omega_{\mathcal{K}}^{1}, i_{+1}(\Omega(n, p))\right)$ such that

(i) $\psi_{0}=\psi$,

(ii) $\psi_{\lambda}|L=\psi| L$,

(iii) $\operatorname{pr}_{p+1}\left(\pi_{1}^{k} \circ \psi_{1}(u)\left(x_{1}, \cdots, x_{n+1}\right)\right)=x_{n+1}$ for any $u \in K$. 
Proof Let us define e: $K \rightarrow \mathbb{R}^{p+1}$ by $\mathbf{e}(u)=\left(\pi_{1}^{k} \circ \psi(u)\right)\left(\mathbf{e}_{n+1}\right)$. Since $\psi(L) \subset$ $i_{+1}(\Omega(n, p))$, we have that, for any $u \in L, \mathbf{e}(u)=\mathbf{e}_{p+1}$. Consider the fiber bundle $\mathfrak{d}_{\mathbb{R}^{p+1}}: J^{k}(n+1, p+1) \rightarrow \mathbb{R}^{p+1}$ defined by $\mathfrak{d}_{\mathbb{R}^{p+1}}\left(j_{\mathbf{0}}^{k} f\right)=j_{\mathbf{0}}{ }^{1} f\left(\mathbf{e}_{n+1}\right)$. Since $\operatorname{dim} K<p, K \backslash L$ is a manifold, $\psi \mid(K \backslash L)$ is smooth and since $\Omega_{\mathcal{K}}^{1}$ is an open subset, it follows from the transversality theorem and the covering homotopy property of $\mathfrak{d}_{\mathbb{R}^{p+1}}$ that there exists a homotopy $\varphi_{\lambda}: K \rightarrow J^{k}(n+1, p+1)$ relative to $L$ with $\varphi_{0}=\psi$ such that

(1) the deformation $\mathbf{u}_{\lambda}=\mathfrak{d}_{\mathbb{R}^{p+1}} \circ \varphi_{\lambda}$ of $\mathbf{e}$ with $\mathbf{u}_{0}=\mathbf{e}$ satisfies that $\mathbf{u}_{1}$ does not take the value of any nonpositive multiple of $\mathbf{e}_{p+1}$,

(2) $\varphi_{\lambda}(K) \subset \Omega_{\mathcal{K}}^{1}$ for any $\lambda$.

In the following an element of $G L(m)$ is regarded as a linear isomorphism of $\mathbb{R}^{m}$ and $E_{m}$ is the unit matrix of degree $m$. Let $h_{\lambda}^{1}:(K, L) \rightarrow\left(G L(p+1), E_{p+1}\right)$ be the homotopy defined by $h_{\lambda}^{1}(u)=\left((1-\lambda)+\lambda /\left\|\mathbf{u}_{1}(u)\right\|\right) E_{p+1}$. It follows from (1) that $h_{1}^{1}(u)\left(\mathbf{u}_{1}(u)\right) \in S^{p}$ and $h_{1}^{1}(u)\left(\mathbf{u}_{1}(u)\right) \neq-\mathbf{e}_{p+1}$ for any $u \in K$. By considering the rotation which is the identity on all points orthogonal to both $\mathbf{u}_{1}(u)$ and $\mathbf{e}_{p+1}$ and rotates the great circle through $\mathbf{u}_{1}(u)$ and $\mathbf{e}_{p+1}$ so as to carry $\mathbf{u}_{1}(u)$ to $\mathbf{e}_{p+1}$ along the shorter way (when $\mathbf{u}_{1}(u)=\mathbf{e}_{p+1}$, we consider $E_{p+1}$ ), we have the homotopy $h_{\lambda}^{2}:(K, L) \rightarrow\left(S O(p+1), E_{p+1}\right)$ relative to $L$ such that $h_{0}^{2}(u)=E_{p+1}$ and $h_{1}^{2}(u) \circ$ $h_{1}^{1}(u)\left(\mathbf{u}_{1}(u)\right)=\mathbf{e}_{p+1}$ for any $u \in K$. Let $h_{\lambda}:(K, L) \rightarrow\left(G L(p+1), E_{p+1}\right)$ be the homotopy defined by $h_{\lambda}=h_{2 \lambda}^{1}$ for $0 \leqq \lambda \leqq 1 / 2$ and $h_{\lambda}=h_{2 \lambda-1}^{2} \circ h_{1}^{1}$ for $1 / 2 \leqq \lambda \leqq 1$. Define $\kappa_{p+1}: K \rightarrow J^{1}(n+1,1)$ by

$$
\kappa_{p+1}(u)=\operatorname{pr}_{p+1} \circ \pi_{1}^{k}\left(j^{k}\left(h_{1}(u)\right) \circ \varphi_{1}(u)\right) .
$$

Since $\kappa_{p+1}(u)$ is of rank 1 for any $u \in K$, we have the unique vector $V(u) \in \mathbb{R}^{n+1}$ of length 1 such that $V(u)$ is perpendicular to $\operatorname{Ker}\left(\kappa_{p+1}(u)\right)$ and $\kappa_{p+1}(u)(V(u))$ is positive. Namely, $h_{1}(u) \circ \pi_{1}^{k}\left(\varphi_{1}(u)\right)(V(u))$ is directed to the same orientation of $\mathbf{e}_{p+1}$. Since $\kappa_{p+1}(u)\left(\mathbf{e}_{n+1}\right)=1, V(u)$ cannot be equal to $-\mathbf{e}_{n+1}$.

We set $v(u)=\kappa_{p+1}(u)(V(u))>0$. By considering the rotation which is the identity on all points orthogonal to both $V(u)$ and $\mathbf{e}_{n+1}$ and rotates the great circle through $V(u)$ and $\mathbf{e}_{n+1}$ so as to carry $\mathbf{e}_{n+1}$ to $V(u)$ along the shorter way, we again have the homotopy $H_{\lambda}^{1}:(K, L) \rightarrow\left(S O(n+1), E_{n+1}\right)$ relative to $L$ such that $H_{0}^{1}(u)=E_{n+1}$ and $H_{1}^{1}(u)\left(\mathbf{e}_{n+1}\right)=V(u)$ for any $u \in K$. Let $H_{\lambda}^{2}:(K, L) \rightarrow\left(G L(n+1), E_{n+1}\right)$ be the homotopy relative to $L$ defined by $H_{\lambda}^{2}(u)=((1-\lambda)+\lambda / v(u)) E_{n+1}$. Let $H_{\lambda}:(K, L) \rightarrow\left(G L(n+1), E_{n+1}\right)$ be the homotopy defined by $H_{\lambda}(u)=H_{2 \lambda}^{1}(u)$ for $0 \leqq \lambda \leqq 1 / 2$ and $H_{\lambda}(u)=H_{2 \lambda-1}^{2}(u) \circ H_{1}^{1}(u)$ for $1 / 2 \leqq \lambda \leqq 1$. Then we have that 
for any $u \in K$,

$$
\begin{aligned}
\kappa_{p+1}(u) \circ H_{1}(u)\left(\mathbf{e}_{n+1}\right) & =\kappa_{p+1}(u) \circ H_{1}^{2}(u) \circ H_{1}^{1}(u)\left(\mathbf{e}_{n+1}\right) \\
& =\kappa_{p+1}(u) \circ H_{1}^{2}(u)(V(u)) \\
& =\kappa_{p+1}(u)(V(u)) / v(u) \\
& =1 .
\end{aligned}
$$

Since $H_{1}^{1}(u) \in S O(n+1)$ and $\mathbf{e}_{i}$ is orthogonal to $\mathbf{e}_{n+1}(i<n+1), H_{1}^{1}(u)\left(\mathbf{e}_{i}\right)$ is orthogonal to $H_{1}^{1}(u)\left(\mathbf{e}_{n+1}\right)=V(u)$. Namely, $H_{1}(u)\left(\mathbf{e}_{i}\right)$ lies in $\operatorname{Ker}\left(\kappa_{p+1}(u)\right)$. Hence, we have

$$
\kappa_{p+1}(u) \circ H_{1}(u)\left(\mathbf{e}_{i}\right)=0 \quad \text { for } i<n+1 .
$$

Define the homotopy $\psi_{\lambda}:(K, L) \rightarrow\left(\Omega_{\mathcal{K}}^{1}, i_{+1}(\Omega(n, p))\right.$ relative to $L$ by

$$
\psi_{\lambda}(u)= \begin{cases}\varphi_{3 \lambda}(u) & \text { for } 0 \leqq \lambda \leqq 1 / 3 \\ h_{3 \lambda-1}(u) \circ \varphi_{1}(u) & \text { for } 1 / 3 \leqq \lambda \leqq 2 / 3 \\ h_{1}(u) \circ \varphi_{1}(u) \circ H_{3 \lambda-2}(u) & \text { for } 2 / 3 \leqq \lambda \leqq 1\end{cases}
$$

By the definition we have

$$
\operatorname{pr}_{p+1} \circ \pi_{1}^{k}\left(\psi_{1}(u)\right)\left(\mathbf{e}_{i}\right)=\left\{\begin{array}{l}
0 \text { for } i<n+1 \\
1 \text { for } i=n+1
\end{array}\right.
$$

This is what we want.

Proposition 6.2 Under the same assumption of Lemma 6.1, we have a homotopy $\Psi_{\lambda}:(K, L) \rightarrow\left(\Omega_{\mathcal{K}}^{1}, i_{+1}(\Omega(n, p))\right)$ such that

(i) $\Psi_{0}=\psi$,

(ii) $\Psi_{\lambda}|L=\psi| L$,

(iii) $\Psi_{1}(K) \subset i_{+1}(\Omega(n, p))$.

Proof Let $\psi_{\lambda}$ be the homotopy given in Lemma 6.1. Let us express $\psi_{\lambda}(u)=$ $\left(f_{\lambda}^{1}(u), f_{\lambda}^{2}(u), \cdots, f_{\lambda}^{p+1}(u)\right)$ using the coordinates of $\mathbb{R}^{p+1}$, where $f_{\lambda}^{i}(u)$ is regarded as a polynomial of degree at most $k$ with constant 0 . We note that

$$
f_{1}^{p+1}(u)\left(x_{1}, \cdots, x_{n+1}\right)=x_{n+1}+\text { higher term. }
$$

Let $\operatorname{Diff}\left(\mathbb{R}^{n+1}, 0\right)$ be the space of all germs of local diffeomorphisms of $\left(\mathbb{R}^{n+1}, 0\right)$. Let us define a homotopy of maps $\Phi_{\lambda}:(K, L) \rightarrow\left(\operatorname{Diff}\left(\mathbb{R}^{n+1}, 0\right), \mathrm{id}_{\left(\mathbb{R}^{n+1}, 0\right)}\right)$ by

$$
\Phi_{\lambda}(u)\left(x_{1}, \cdots, x_{n+1}\right)=\left(x_{1}, \cdots, x_{n+1}+\lambda\left(f_{1}^{p+1}(u)-x_{n+1}\right)\right) .
$$


It is obvious that $\Phi_{\lambda}(u)$ is a germ of a diffeomorphism of $\left(\mathbb{R}^{n+1}, 0\right)$. Then we have the inverse $\Phi_{\lambda}(u)^{-1}$ such that

$$
\operatorname{pr}_{p+1} \circ \psi_{1}(u) \circ \Phi_{1}(u)^{-1}\left(x_{1}, \cdots, x_{n+1}\right)=x_{n+1} .
$$

We now define $\phi_{\lambda}:(K, L) \rightarrow\left(\Omega_{\mathcal{K}}^{1}, i_{+1}(\Omega(n, p))\right)$ by

$$
\phi_{\lambda}(u)=\psi_{1} \circ j_{0}^{k}\left(\Phi_{\lambda}(u)^{-1}\right)
$$

In order to exclude the terms containing $x_{n+1}$ from $y_{j} \circ \phi_{1}(1 \leqq j \leqq p)$ we define the homotopy $\eta_{\lambda}:(K, L) \rightarrow\left(J^{k}(n+1, p+1), i_{+1}\left(J^{k}(n, p)\right)\right)$ by

$$
\begin{aligned}
\eta_{\lambda}(u)(x)=(1 & -\lambda) \phi_{1}(u)\left(x_{1}, \cdots, x_{n+1}\right) \\
& +\lambda\left(\phi_{1}(u)\left(x_{1}, \cdots, x_{n}, 0\right)+\left(0, \cdots, 0, x_{n+1}\right)\right) .
\end{aligned}
$$

It is obvious that $\eta_{1}(K) \subset i_{+1}(\Omega(n, p))$ and that $\eta_{\lambda}|L=\psi| L$. It remains to prove that $\eta_{\lambda}$ is a homotopy to $\Omega_{\mathcal{K}}^{1}$. It follows from (6-1) and (6-2) that

$$
\operatorname{pr}_{p+1} \circ \eta_{\lambda}(u)(x)=(1-\lambda)\left(x_{n+1}\right)+\lambda x_{n+1}=x_{n+1} \text {. }
$$

Let us express $\eta_{\lambda}(u)=\left(g_{\lambda}^{1}(u), g_{\lambda}^{2}(u), \cdots, g_{\lambda}^{p+1}(u)\right)$, where $g_{\lambda}^{i}(u)$ is regarded as a polynomial of degree at most $k$ with constant 0 . Consider the ideal $\mathfrak{I}_{\lambda}(u)$ generated by $g_{\lambda}^{1}(u), g_{\lambda}^{2}(u), \cdots, g_{\lambda}^{p+1}(u)$ in $\mathfrak{m}_{n+1} / \mathfrak{m}_{n+1}^{k+1}$. Then $\mathfrak{I}_{\lambda}(u)$ is constantly equal to $\mathfrak{I}_{0}(u)$, and hence $Q\left(\eta_{\lambda}(u)\right) \approx Q\left(\psi_{1}(u)\right)$. Since $\psi_{1}(u) \in \Omega_{\mathcal{K}}^{1}$, we have $\eta_{\lambda}(u) \in \Omega_{\mathcal{K}}^{1}$ by [38]. Then the required homotopy $\Psi_{\lambda}$ is defined by $\Psi_{\lambda}=\psi_{3 \lambda}(0 \leqq \lambda \leqq 1 / 3)$, $\Psi_{\lambda}=\phi_{3 \lambda-1}(1 / 3 \leqq \lambda \leqq 2 / 3)$ and $\Psi_{\lambda}=\eta_{3 \lambda-2}(2 / 3 \leqq \lambda \leqq 1)$.

Proposition 6.3 Let $\Omega(n, p)$ be a $\mathcal{K}$-invariant open subset of $J^{k}(n, p)$. Then

$$
i_{+1}: \Omega(n, p) \rightarrow \Omega_{\mathcal{K}}^{1}
$$

is a homotopy $(p-1)$-equivalence.

Proof Let $\iota_{n}$ denote a jet of $\Omega(n, p)$ and $\iota_{n+1}=i_{+1}\left(\iota_{n}\right)$. We first prove that $\left(i_{+1}\right)_{*}: \pi_{i}(\Omega(n, p)) \rightarrow \pi_{i}\left(\Omega_{\mathcal{K}}^{1}\right)$ is surjective for $0 \leqq i \leqq p-1$. Indeed, let $[a] \in \pi_{i}\left(\Omega_{\mathcal{K}}^{1}\right)$ be represented by $a:\left(S^{i}, \mathbf{e}_{1}\right) \rightarrow\left(\Omega_{\mathcal{K}}^{1}, \iota_{n+1}\right)$. Then by Proposition 6.2 we have a homotopy $\varphi_{\lambda}:\left(S^{i}, \mathbf{e}_{1}\right) \rightarrow\left(\Omega_{\mathcal{K}}^{1}, \iota_{n+1}\right)$ such that $\varphi_{1}\left(S^{i}\right) \subset i_{+1}(\Omega(n, p))$.

Next let $0 \leqq i<p-1$. Let $[b] \in \pi_{i}(\Omega(n, p))$ be represented by $b:\left(S^{i}, \mathbf{e}_{1}\right) \rightarrow$ $\left(\Omega(n, p), \iota_{n}\right)$ such that $\left(i_{+1}\right)_{*}([b])=0$. Then we have a homotopy $\tilde{\varphi}: S^{i} \times[0,1] \rightarrow$ $\left(\Omega_{\mathcal{K}}^{1}, \iota_{n+1}\right)$ such that $\tilde{\varphi} \mid S^{i} \times 0=i_{+1} \circ b$ under the identification $S^{i}=S^{i} \times 0$ and $\tilde{\varphi}\left(\mathbf{e}_{1} \times[0,1] \cup S^{i} \times 1\right)=\iota_{n+1}$. It follows from Proposition 6.2 that since $i+1<p$, there exists a homotopy $\Phi_{\lambda}:\left(S^{i} \times[0,1], S^{i} \times 0 \cup \mathbf{e}_{1} \times[0,1] \cup S^{i} \times 1\right) \rightarrow\left(\Omega_{\mathcal{K}}^{1}, i_{+1}(\Omega(n, p))\right)$ 
relative to $S^{i} \times 0 \cup \mathbf{e}_{1} \times[0,1] \cup S^{i} \times 1$ such that $\Phi_{1}\left(S^{i} \times[0,1]\right) \subset i_{+1}(\Omega(n, p))$. This proves the injectivity of $\left(i_{+1}\right)_{*}: \pi_{i}(\Omega(n, p)) \rightarrow \pi_{i}\left(\Omega_{\mathcal{K}}^{1}\right)$.

\section{Proof of Theorem 1.2}

Let $A_{m, q}$ express $G_{m, q}$ or $\widetilde{G}_{m, q}$. Let $i_{A_{m+r}}: A_{m, q} \rightarrow A_{m+r, q}$ denote the injection mapping an $m$-plane $a$ to the $(m+r)$-plane including $a$ and the canonical vectors $\mathbf{e}_{q+m+1}, \cdots, \mathbf{e}_{q+m+r}$ in $\mathbb{R}^{q+m+r}$. We use the notation

$$
\boldsymbol{\Omega}_{\mathcal{K}}^{m}=\Omega_{\mathcal{K}}\left(\gamma_{G_{n+m}}^{n+m}, T P \oplus \varepsilon_{P}^{m}\right) .
$$

Lemma 7.1 The map $i_{A_{m+r}}: A_{m, q} \rightarrow A_{m+r, q}$ is a homotopy $m$-equivalence.

Proof We only prove the unoriented case. The proof in the oriented case is similar. Let us consider the diagram with the canonical maps as described

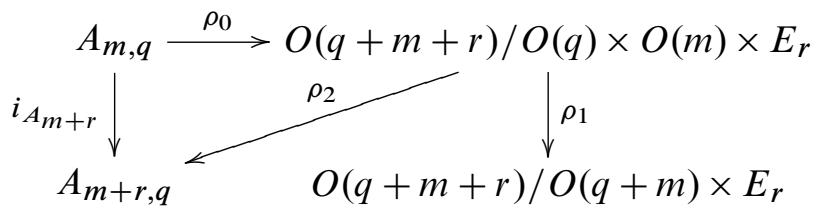

where $\rho_{0}$ is induced from the inclusion $\mathbb{R}^{q+m}=\mathbb{R}^{q+m} \times 0 \rightarrow \mathbb{R}^{q+m+r}$ and $\rho_{1}$ and $\rho_{2}$ are induced from the inclusions $O(q) \times O(m) \rightarrow O(q+m)$ and $O(m) \times E_{r} \rightarrow O(m+r)$ respectively. Since $A_{m, q}$ is a fiber of the fiber bundle $\rho_{1}, \rho_{0}$ is a homotopy $(q+m-1)-$ equivalence. Since $\rho_{2}$ is a homotopy $m$-equivalence, $i_{A_{m+r}}$ is also a homotopy $m-$ equivalence.

In the following lemma $p$ is not necessarily larger than $n$.

Lemma 7.2 Let $\Omega(n, p)$ be a $\mathcal{K}$-invariant open subset of $J^{k}(n, p)$. Then the fiber map $\Delta^{\left(\Omega, \Omega_{\mathcal{K}}\right)}: \boldsymbol{\Omega} \rightarrow \boldsymbol{\Omega}_{\mathcal{K}}^{1}$ is a homotopy $\min \{n, p-1\}$-equivalence.

Proof Consider the commutative diagram

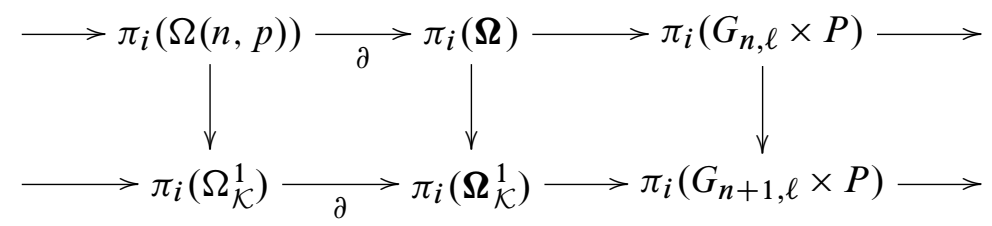

which is induced from the homomorphisms $\left(\Delta^{\left(\Omega, \Omega_{\mathcal{K}}\right)}\right)_{*}$ of the exact sequence of the homotopy groups for the fiber bundle $\boldsymbol{\Omega}$ over $G_{n, \ell} \times P$ to that for the fiber bundle $\boldsymbol{\Omega}_{\mathcal{K}}^{1}$ 
over $G_{n+1, \ell} \times P$. Then it follows from Lemma 7.1 for $\left(i^{G}\right)_{*}$ and Proposition 6.3 that if $0 \leqq i<\min \{n, p-1\}$, then $\left(\Delta^{\left(\Omega, \Omega_{\mathcal{K}}\right)}\right)_{*}: \pi_{i}(\boldsymbol{\Omega}) \rightarrow \pi_{i}\left(\boldsymbol{\Omega}_{\mathcal{K}}^{1}\right)$ is an isomorphism by the five lemma and if $i=\min \{n, p-1\}$, then it is an epimorphism by [15, Lemma 3.2].

We are now ready to prove Theorem 1.2.

Proof of Theorem 1.2 We only prove the unoriented case. In the oriented case we only need the Thom Isomorphism Theorem under the coefficient group $\mathbb{Z}$. It follows from Lemma 7.2 and the Whitehead theorem [53, Section 5, 9 Theorem] that

$$
\left(\Delta^{\left(\Omega, \Omega_{\mathcal{K}}\right)}\right)_{*}: H_{i}(\boldsymbol{\Omega}) \rightarrow H_{i}\left(\boldsymbol{\Omega}_{\mathcal{K}}^{1}\right)
$$

is an isomorphism for $0 \leqq i<n$ and an epimorphism for $i=n$.

Let $\ell \gg n, p$. By virtue of the Thom Isomorphism Theorem, we have that

$$
T\left(\mathbf{b}(\hat{\gamma})^{\left(\Omega, \Omega_{\mathcal{K}}\right)}\right)_{*}: H_{i+\ell}\left(T\left(\hat{\gamma}_{\mathbf{\Omega}}^{\ell}\right) ; \mathbb{Z} /(2)\right) \longrightarrow H_{i+\ell}\left(T\left(\hat{\gamma}_{\mathbf{\Omega}_{\mathcal{K}}^{1}}^{\ell}\right) ; \mathbb{Z} /(2)\right)
$$

is an isomorphism for $-\ell \leqq i<n$ and an epimorphism for $i=n$.

Let $\mathcal{C}$ denote the Serre class of finite groups of orders prime to two. Then it follows from [50, Proposition 2, page 277] that

$$
T\left(\mathbf{b}(\widehat{\gamma})^{\left(\Omega, \Omega_{\mathcal{K}}\right)}\right)_{*}: H_{i+\ell}\left(T\left(\hat{\gamma}_{\mathbf{\Omega}}^{\ell}\right) ; \mathbb{Z}\right) \longrightarrow H_{i+\ell}\left(T\left(\hat{\gamma}_{\mathbf{\Omega}_{\mathcal{K}}^{1}}^{\ell}\right) ; \mathbb{Z}\right)
$$

is a $\mathcal{C}$-isomorphism for $-\ell \leqq i<n$ and a $\mathcal{C}$-epimorphism for $i=n$. By the Whitehead theorem modulo $\mathcal{C}$ [50, Theorem 3, page 276],

$$
T\left(\mathbf{b}(\hat{\gamma})^{\left(\Omega, \Omega_{\mathcal{K}}\right)}\right)_{*}: \pi_{i+\ell}\left(T\left(\hat{\gamma}_{\mathbf{\Omega}}^{\ell}\right)\right) \longrightarrow \pi_{i+\ell}\left(T\left(\hat{\gamma}_{\mathbf{\Omega}_{\mathcal{K}}^{\ell}}^{\ell}\right)\right)
$$

is a $\mathcal{C}$-isomorphism for $-\ell \leqq i<n$ and a $\mathcal{C}$-epimorphism for $i=n$.

Let $\mathfrak{N}^{i}(\boldsymbol{\Omega})$ (respectively $\mathfrak{N}^{i}\left(\boldsymbol{\Omega}_{\mathcal{K}}^{1}\right)$ ) denote the cobordism group of smooth maps $s$ of closed $i$-manifolds $M$ to $\boldsymbol{\Omega}$ (respectively $\boldsymbol{\Omega}_{\mathcal{K}}^{1}$ ) under the corresponding cobordism of smooth maps such that there exists a bundle map of the stable $\ell$-dimensional normal bundle $v_{M}$ to $\hat{\gamma}_{\boldsymbol{\Omega}}^{\ell}$ (respectively $\hat{\gamma}_{\mathbf{\Omega}_{\mathcal{K}}^{\ell}}^{\ell}$ ) covering $s$. It follows from the standard argument in the cobordism theory (see, for example, Stong [55]) that

$$
\mathfrak{N}^{i}(\boldsymbol{\Omega}) \approx \pi_{i+\ell}\left(T\left(\hat{\gamma}_{\boldsymbol{\Omega}}^{\ell}\right)\right) \text { and } \mathfrak{N}^{i}\left(\boldsymbol{\Omega}_{\mathcal{K}}^{1}\right) \approx \pi_{i+\ell}\left(T\left(\hat{\gamma}_{\boldsymbol{\Omega}_{\mathcal{K}}^{1}}^{\ell}\right)\right) .
$$

Consequently, any element of $\pi_{i+\ell}\left(T\left(\hat{\gamma}_{\mathbf{\Omega}}^{\ell}\right)\right)$ and $\pi_{i+\ell}\left(T\left(\hat{\gamma}_{\boldsymbol{\Omega}_{\mathcal{K}}^{1}}^{\ell}\right)\right)$ is of order two. This together with (7-1) proves Theorem 1.2.

By Lemmas 5.2 and 5.4 it will be easy to see inductively that $\Omega_{\mathcal{K}}(n+m, p+m)$ is the subset of $J^{k}(n+m, p+m)$ which consists of all $\mathcal{K}$-orbits $\mathcal{K}\left(i_{+1}(z)\right)$ for $k$-jets $z \in \Omega_{\mathcal{K}}(n+m-1, p+m-1)$ for $m>1$ and that if $\Omega(n, p)=\Omega^{I}(n, p) \neq \varnothing$, then we 
have $\Omega^{I}(n+m, p+m)=\Omega_{\mathcal{K}}(n+m, p+m)$. By using the map $J^{k}(n+m, p+m) \rightarrow$ $J^{k}(n+m+q, p+m+q)$ sending a jet $j_{0}^{k} f$ to $j_{0}^{k}\left(f \times \mathrm{id}_{\mathbb{R}^{q}}\right)$, we have the canonical map $\Delta^{\left(\Omega_{\mathcal{K}}^{m}, \Omega_{\mathcal{K}}^{m+q}\right)}: \boldsymbol{\Omega}_{\mathcal{K}}^{m} \rightarrow \boldsymbol{\Omega}_{\mathcal{K}}^{m+q}$. This induces the canonical bundle map

$$
\mathbf{b}(\widehat{\gamma})^{\left(\Omega_{\mathcal{K}}^{m}, \Omega_{\mathcal{K}}^{m+q}\right)}: \hat{\gamma}_{\boldsymbol{\Omega}_{\mathcal{K}}^{m}}^{\ell} \rightarrow \hat{\gamma}_{\boldsymbol{\Omega}_{\mathcal{K}}^{m+q}}^{\ell}
$$

and the associated map

$$
T\left(\mathbf{b}(\widehat{\gamma})^{\left(\Omega_{\mathcal{K}}^{m}, \Omega_{\mathcal{K}}^{m+q}\right)}\right): T\left(\gamma_{\mathbf{\Omega}_{\mathcal{K}}^{m}}^{\ell}\right) \rightarrow T\left(\hat{\gamma}_{\mathbf{\Omega}_{\mathcal{K}}^{\ell+q}}^{\ell}\right) .
$$

Let $\theta$ denote an integer such that $\theta=1$ when $n<p$ and $\theta=n+2-p$ when $n \geqq p \geqq 2$.

Proposition 7.3 Let $\theta$ be the integer as above. Then the homomorphism

$$
\lim _{\ell \rightarrow \infty} \pi_{n+\ell}\left(T\left(\hat{\gamma}_{\boldsymbol{\Omega}_{\mathcal{K}}^{\ell}}^{\ell}\right)\right) \longrightarrow \lim _{\ell \rightarrow \infty} \pi_{n+\ell}\left(T\left(\hat{\gamma}_{\boldsymbol{\Omega}_{\mathcal{K}}^{\theta+q}}^{\ell}\right)\right)
$$

induced from the above map $T\left(\mathbf{b}(\widehat{\gamma})^{\left(\Omega_{\mathcal{K}}^{\theta}, \Omega_{\mathcal{K}}^{\theta+q}\right)}\right)$ is an isomorphism for $q \geqq 0$.

Proof We only prove the unoriented case. The proof proceeds as in the Proof of Theorem 1.2. By the iterated use of Lemma 7.2, we have that $T\left(\mathbf{b}(\widehat{\gamma})^{\left(\Omega_{\mathcal{K}}^{\theta}, \Omega_{\mathcal{K}}^{\theta+q}\right)}\right)$ is a homotopy $\min \{n+\theta, p+\theta-1\}$-equivalence, and hence, a homotopy $(n+1)-$ equivalence. Therefore,

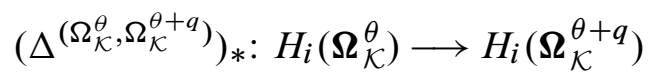

is an isomorphism for $0 \leqq i \leqq n$ and an epimorphism for $i=n+1$. By the argument similar to that in the proof of Theorem 1.2, we have that

$$
\left(T\left(\mathbf{b}(\hat{\gamma})^{\left(\Omega_{\mathcal{K}}^{\theta}, \Omega_{\mathcal{K}}^{\theta+q}\right)}\right)\right)_{*}: \pi_{i+\ell}\left(T\left(\hat{\gamma}_{\mathbf{\Omega}_{\mathcal{K}}^{\ell}}^{\ell}\right)\right) \longrightarrow \pi_{i+\ell}\left(T\left(\hat{\gamma}_{\boldsymbol{\Omega}_{\mathcal{K}}^{\theta+q}}^{\ell}\right)\right)
$$

is a $\mathcal{C}$-isomorphism for $-\ell \leqq i \leqq n$ and a $\mathcal{C}$-epimorphism for $i=n+1$. Since any element of the groups in (7-2) is of order two, we obtain the proposition.

\section{Classifying space}

In this section we will induce the classifying space $B_{\mathfrak{O}}$ in Theorem 1.3. We assume that $P$ is oriented in the oriented case.

Consider the vector bundle $J^{k}\left(\gamma_{G_{n+m}}^{n+m}, \mathbb{R}^{p+m}\right)$ with the projection $\pi_{G_{n+m}}^{k}$ onto

$$
G_{n+m}:=G_{n+m} \times\{\text { a point }\}
$$

and the open subbundle $\Omega_{\mathcal{K}}\left(\gamma_{G_{n+m}}^{n+m}, \mathbb{R}^{p+m}\right)$ of $J^{k}\left(\gamma_{G_{n+m}}^{n+m}, \mathbb{R}^{p+m}\right)$ associated to $\Omega_{\mathcal{K}}^{m}$, where $\mathbb{R}^{p+m}$ is regarded as a vector bundle over a point. As in Section 3, the spaces $\left\{T\left(\left.\left(\pi_{G_{n+m}}^{k}\right)^{*}\left(\hat{\gamma}_{G_{n+m}^{\ell}}^{\ell}\right)\right|_{\boldsymbol{\Omega}_{\mathcal{K}}^{m}}\right)\right\}_{\ell}$ constitute a spectrum. 
Let $\varkappa$ denote any integer with $\varkappa \geqq p+3$. Let $\ell \gg n, p, \varkappa$. We set $\mathbb{G}_{n+\theta+\varkappa}=$ $A_{n+\theta+\varkappa, \ell+p}$. Let $P$ be embedded in $\mathbb{R}^{p+\varkappa}$. Let $x \in G_{n+\theta}, y \in P$ and $\left(v_{P}\right)_{y}$ be the orthogonal complement of $T_{y} P$ in $\mathbb{R}^{p+\varkappa}$. Let $c: G_{n+\theta} \times P \rightarrow \mathbb{G}_{n+\theta+\varkappa}$ denote the map such that $c(x, y)$ is the $(n+\theta+\varkappa)$-subspace $x \oplus\left(v_{P}\right)_{y}$ in $\mathbb{R}^{n+\theta+\varkappa+\ell+p}$. Let c: $\pi_{G_{n+\theta}}^{*}\left(\gamma_{G_{n+\theta}}^{n+\theta}\right) \oplus \pi_{P}^{*}\left(v_{P}\right) \rightarrow \gamma_{\mathbb{G}_{n+\theta+\varkappa}}^{n+\theta+\varkappa}$ be the bundle map which is canonically induced to cover the classifying map $c$.

Let $\mathbf{J}^{k}\left(\gamma^{n+\theta} \oplus v, T P \oplus \varepsilon_{P}^{\theta} \oplus v_{P}\right)$ denote

$$
\operatorname{Hom}\left(\bigoplus_{i=1}^{k} S^{i}\left(\pi_{G_{n+\theta}}^{*}\left(\gamma_{G_{n+\theta}}^{n+\theta}\right) \oplus \pi_{P}^{*}\left(v_{P}\right)\right), \pi_{P}^{*}\left(T P \oplus \varepsilon_{P}^{\theta} \oplus v_{P}\right)\right)
$$

and define the fiber map

$$
J^{k}\left(\gamma_{G_{n+\theta}}^{n+\theta}, T P \oplus \varepsilon_{P}^{\theta}\right) \longrightarrow \mathbf{J}^{k}\left(\gamma^{n+\theta} \oplus v, T P \oplus \varepsilon_{P}^{\theta} \oplus v_{P}\right)
$$

over $G_{n+\theta} \times P$ by mapping $j_{x}^{k} \alpha \in J_{x, y}^{k}\left(\gamma_{G_{n+\theta}+\theta}^{n+\theta}, T P \oplus \varepsilon_{P}^{\theta}\right)$ to $j_{(x, y)}^{k}\left(\alpha \times \operatorname{id}_{\left(v_{P}\right)_{y}}\right)$. We also obtain the bundle map

$$
\mathbf{J}^{k}\left(\gamma^{n+\theta} \oplus v, T P \oplus \varepsilon_{P}^{\theta} \oplus v_{P}\right) \longrightarrow J^{k}\left(\gamma_{\mathbb{G}_{n+\theta+\varkappa}}^{n+\theta+\varkappa}, T P \oplus \varepsilon_{P}^{\theta} \oplus v_{P}\right)
$$

covering $\left(c, \pi_{P}\right): G_{n+\theta} \times P \rightarrow \mathbb{G}_{n+\theta+\varkappa} \times P$ which is canonically induced from the bundle map $\mathbf{c}$ and $\operatorname{id}_{T P \oplus \varepsilon_{P}^{\theta} \oplus v_{P}}$. It follows from Lemma 7.1 that $\left(c, \pi_{P}\right)$ is a homotopy $(n+\theta)$-equivalence. The composite of the maps in (8-1) and (8-2) on the fibers over $(x, y)$ and $(c(x, y), y)$ induces a map

$$
\Omega_{\mathcal{K}}\left(\left(\gamma_{G_{n+\theta}}^{n+\theta}\right)_{x}, T_{y} P \oplus \varepsilon_{y}^{\theta}\right) \longrightarrow \Omega_{\mathcal{K}}\left(\left(\gamma_{\mathbb{G}_{n+\theta+\varkappa}+\theta+\varkappa}^{n+\theta}\right)_{c(x, y)}, T_{y} P \oplus \varepsilon_{y}^{\theta} \oplus\left(v_{P}\right)_{y}\right),
$$

which is a homotopy $(n+1)$-equivalence by the iterated use of Proposition 6.3 and $G_{n+\theta} \times P \rightarrow \mathbb{G}_{n+\theta+\varkappa} \times P$ is a homotopy $(n+\theta)$-equivalence by Lemma 7.1. Consequently, we obtain the fiber map

$$
\mathbf{j}_{\Omega_{\mathcal{K}}}: \Omega_{\mathcal{K}}\left(\gamma_{G_{n+\theta}}^{n+\theta}, T P \oplus \varepsilon_{P}^{\theta}\right) \longrightarrow \Omega_{\mathcal{K}}\left(\gamma_{\mathbb{G}_{n+\theta+\varkappa}}^{n+\theta+\varkappa}, T P \oplus \varepsilon_{P}^{\theta} \oplus v_{P}\right)
$$

covering $\left(c, \pi_{P}\right)$, which is also a homotopy $(n+1)$-equivalence.

Trivialization $T P \oplus v_{P} \rightarrow \varepsilon_{P}^{p+\varkappa}$ induces the bundle isomorphism

$$
J^{k}\left(\gamma_{\mathbb{G}_{n+\theta+\varkappa}}^{n+\theta+\varkappa}, T P \oplus \varepsilon_{P}^{\theta} \oplus v_{P}\right) \longrightarrow J^{k}\left(\gamma_{\mathbb{G}_{n+\theta+\varkappa}}^{n+\theta+\varkappa}, \mathbb{R}^{p+\theta+\varkappa}\right) \times P
$$

over $\mathbb{G}_{n+\theta+\varkappa} \times P$, where $\mathbb{R}^{p+\theta+\varkappa}$ is regarded as the trivial vector bundle over a point. Let $\Omega_{\mathcal{K}}\left(\gamma_{\mathbb{G}_{n+\theta+\varkappa}}^{n+\theta+\varkappa}, \mathbb{R}^{p+\theta+\varkappa}\right)$ be the open subbundle associated to $\Omega_{\mathcal{K}}^{\theta+\varkappa}$. Then we have the bundle map

(8-4) $\quad \mathbf{k}_{\Omega_{\mathcal{K}}}: \Omega_{\mathcal{K}}\left(\gamma_{\mathbb{G}_{n+\theta+\varkappa}}^{n+\theta+\varkappa}, T P \oplus \varepsilon_{P}^{\theta} \oplus v_{P}\right) \longrightarrow \Omega_{\mathcal{K}}\left(\gamma_{\mathbb{G}_{n+\theta+\varkappa}}^{n+\theta+\varkappa}, \mathbb{R}^{p+\theta+\varkappa}\right) \times P$ 
over $\mathbb{G}_{n+\theta+\varkappa} \times P$. In the following we identify the two spaces in (8-4). Thus we have the following lemma.

Lemma 8.1 The fiber map $\mathbf{k}_{\Omega_{\mathcal{K}}} \circ \mathbf{j}_{\Omega_{\mathcal{K}}}$ covering $\left(c, \pi_{P}\right)$ is a homotopy $(n+1)-$ equivalence.

Let

$$
\pi_{\mathbb{G}_{n+\theta+\varkappa}}^{k}: J^{k}\left(\gamma_{\mathbb{G}_{n+\theta+\varkappa}+\theta+\varkappa}^{\left.n+\mathbb{R}^{p+\theta+\varkappa}\right) \rightarrow \mathbb{G}_{n+\theta+\varkappa}}\right.
$$

be the canonical projection. Let $B\left(\widehat{\gamma}_{\mathbb{G}_{n+\theta+\varkappa}^{\ell+p}}^{\ell}, v_{P}\right)_{\Omega_{\mathcal{K}} \times P}$ denote the vector bundle over $\Omega_{\mathcal{K}}\left(\gamma_{\mathbb{G}_{n+\theta+\varkappa}}^{n+\theta+\varkappa}, \mathbb{R}^{p+\theta+\varkappa}\right) \times P$ defined by

$$
B\left(\hat{\gamma}_{\mathbb{G}_{n+\theta+\varkappa}}^{\ell+p}, v_{P}\right)_{\Omega_{\mathcal{K}} \times P}=\left.\left(\pi_{\mathbb{G}_{n+\theta+\varkappa}}^{k}\right)^{*}\left(\hat{\gamma}_{\mathbb{G}_{n+\theta+\varkappa}}^{\ell+p}\right)\right|_{\Omega_{\mathcal{K}}\left(\gamma_{\mathbb{G}_{n+\theta+\varkappa}}^{n+\theta+\varkappa}, \mathbb{R}^{p+\theta+\varkappa}\right)} \times v_{P} .
$$

This satisfies

$$
\begin{aligned}
& T\left(B\left(\widehat{\gamma}_{\mathbb{G}_{n+\theta+\varkappa}+p}^{\ell+\nu_{P}}\right)_{\Omega_{\mathcal{K}} \times P}\right) \\
& =T\left(\left.\left(\pi_{\mathbb{G}_{n+\theta+\varkappa}}^{k}\right)^{*}\left(\hat{\gamma}_{\mathbb{G}_{n+\theta+\varkappa}+p}^{\ell+p}\right)\right|_{\Omega_{\mathcal{K}}\left(\gamma_{\mathbb{G}_{n+\theta+\varkappa}}^{n+\theta+\varkappa}, \mathbb{R}^{p+\theta+\varkappa}\right)}\right) \wedge T\left(v_{P}\right) .
\end{aligned}
$$

Let

$$
\begin{aligned}
&\left.\left(\pi_{G_{n+\theta}}^{k}\right)^{*}\left(\hat{\gamma}_{G_{n+\theta}}^{\ell}\right) \oplus\left(\pi_{P}^{k}\right)^{*}(T P)\right|_{\Omega_{\mathcal{K}}\left(\gamma_{G_{n+\theta}}^{n+\theta}, T P \oplus \varepsilon_{P}^{\theta}\right)} \\
& \longrightarrow\left(\left.\left(\pi_{\mathbb{G}_{n+\theta+\varkappa}}^{k}\right)^{*}\left(\hat{\gamma}_{\mathbb{G}_{n+\theta+\varkappa}^{\ell+p}}\right)\right|_{\Omega_{\mathcal{K}}\left(\gamma_{\mathbb{G}_{n+\theta+\varkappa}}^{n+\theta+\varkappa}, \mathbb{R}^{p+\theta+\varkappa}\right)}\right) \times P
\end{aligned}
$$

be the bundle map covering $\mathbf{k}_{\Omega_{\mathcal{K}}} \circ \mathbf{j} \Omega_{\mathcal{K}}$ mapping

$$
\left(\gamma_{G_{n+\theta}}^{n+\theta}\right)_{x}^{\perp} \oplus T_{y} P \quad \text { to } \quad\left(\left(\gamma_{G_{n+\theta}}^{n+\theta}\right)_{x} \oplus\left(v_{P}\right)_{y}\right)^{\perp}
$$

where $\perp$ denotes the orthogonal complement. This induces a bundle map

B: $\left.\left(\left(\pi_{G_{n+\theta}}^{k}\right)^{*}\left(\hat{\gamma}_{G_{n+\theta}}^{\ell}\right) \oplus\left(\pi_{P}^{k}\right)^{*}\left(T P \oplus \nu_{P}\right)\right)\right|_{\Omega_{\mathcal{K}}\left(\gamma_{G_{n+\theta}}^{n+\theta}, T P \oplus \varepsilon_{P}^{\theta}\right)}$

$$
\longrightarrow B\left(\widehat{\gamma}_{\mathbb{G}_{n+\theta+\varkappa}^{\ell+p}}^{\ell}, v_{P}\right)_{\Omega_{\mathcal{K}} \times P}
$$

covering

$$
\mathbf{k}_{\Omega_{\mathcal{K}}} \circ \mathbf{j}_{\Omega_{\mathcal{K}}}: \Omega_{\mathcal{K}}\left(\gamma_{G_{n+\theta}}^{n+\theta}, T P \oplus \varepsilon_{P}^{\theta}\right) \rightarrow \Omega_{\mathcal{K}}\left(\gamma_{\mathbb{G}_{n+\theta+\varkappa}}^{n+\theta+\varkappa}, \mathbb{R}^{p+\theta+\varkappa}\right) \times P
$$

which is a bundle map over $P$ and is a homotopy $(n+1)$-equivalence.

Let $X$ and $Y$ be connected polyhedra with base points respectively. Let $\{X ; Y\}$ denote the set of $S$-homotopy classes of $S$-maps. Let $G_{n+\theta+\varkappa}=A_{n+\theta+\varkappa, \ell}$ as in Section 1. 
Proposition 8.2 Let $\Omega(n, p)$ denote a nonempty $\mathcal{K}$-invariant open subset. Let $P$ be oriented in the oriented case. Then there exists an isomorphism

$$
\begin{aligned}
& \lim _{\ell \rightarrow \infty} \pi_{n+\ell}\left(T\left(\hat{\gamma}_{\Omega_{\mathcal{K}}}^{\ell}\left(\gamma_{G_{n+\theta}}^{n+\theta}, T P \oplus \varepsilon_{P}^{\theta}\right)\right)\right) \longrightarrow \\
& \lim _{\ell \rightarrow \infty} \pi_{n+\ell+\varkappa}\left(T\left(\left.\left(\pi_{G_{n+\theta+\varkappa}}^{k}\right)^{*}\left(\widehat{\gamma}_{G_{n+\theta+\varkappa}}^{\ell}\right)\right|_{\Omega_{\mathcal{K}}\left(\gamma_{G_{n+\theta+\varkappa}}^{n+\theta+\varkappa}, \mathbb{R}^{p+\theta+\varkappa}\right)}\right) \wedge T\left(\nu_{P}\right)\right) .
\end{aligned}
$$

Proof Setting $\boldsymbol{\Omega}_{\mathcal{K}}^{\theta}=\Omega_{\mathcal{K}}\left(\gamma_{G_{n+\theta}}^{n+\theta}, T P \oplus \varepsilon_{P}^{\theta}\right)$, we have that

$$
\begin{aligned}
\pi_{n+\ell}\left(T\left(\hat{\gamma}_{\boldsymbol{\Omega}_{\mathcal{K}}^{\ell}}^{\ell}\right)\right) & \approx\left\{S^{n+\ell} ; T\left(\hat{\gamma}_{\boldsymbol{\Omega}_{\mathcal{K}}^{\ell}}^{\ell}\right)\right\} \\
& \approx\left\{S^{n+\ell+p+\varkappa} ; T\left(\hat{\gamma}_{\boldsymbol{\Omega}_{\mathcal{K}}^{\ell}}^{\ell}\right) \wedge S^{p+\varkappa}\right\} \\
& \approx\left\{S^{n+\ell+p+\varkappa} ; T\left(\hat{\gamma}_{\boldsymbol{\Omega}_{\mathcal{K}}^{\ell}} \oplus \varepsilon_{\boldsymbol{\Omega}_{\mathcal{K}}^{\theta}}^{p+\varkappa}\right)\right\} \\
& \approx\left\{S^{n+\ell+p+\varkappa} ; T\left(\left.\hat{\gamma}_{\mathbf{\Omega}_{\mathcal{K}}^{\ell}}^{\theta} \oplus\left(\pi_{P}^{k}\right)^{*}\left(T P \oplus v_{P}\right)\right|_{\mathbf{\Omega}_{\mathcal{K}}^{\theta}}\right)\right\} .
\end{aligned}
$$

By Lemma 8.1 and an argument as in the proof of Theorem $1.2 \mathrm{using}$ the Thom isomorphism Theorem, the associated map $T(\mathbf{B})$ induces the isomorphism between the last group in $(8-5)$ and $\left\{S^{n+\ell+p+\varkappa} ; T\left(B\left(\hat{\gamma}_{\mathbb{G}_{n+\theta+\varkappa}+p}^{\ell+v_{P}}\right)_{\Omega_{\mathcal{K}} \times P}\right)\right\}$. Furthermore, we have that

$$
\begin{aligned}
& \left\{S^{n+\ell+p+\varkappa} ; T\left(B\left(\hat{\gamma}_{\mathbb{G}_{n+\theta+\varkappa}^{\ell+p}}, v_{P}\right)_{\Omega_{\mathcal{K}} \times P}\right)\right\}
\end{aligned}
$$

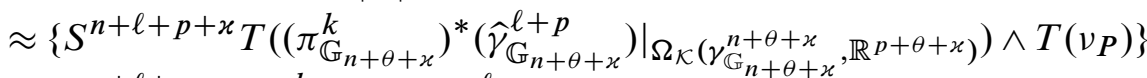

$$
\begin{aligned}
& \approx\left\{S^{n+\ell+\varkappa} ; T\left(\left.\left(\pi_{G_{n+\theta+\varkappa}}^{k}\right)^{*}\left(\widehat{\gamma}_{G_{n+\theta+\varkappa}^{\ell}}^{\ell}\right)\right|_{\Omega_{\mathcal{K}}\left(\gamma_{G_{n+\theta+\varkappa}}^{n+\theta+\varkappa}, \mathbb{R}^{p+\theta+\varkappa}\right)}\right) \wedge T\left(v_{P}\right)\right\} .
\end{aligned}
$$

This proves the proposition.

In the rest of this section we assume that $P$ should be closed and connected. Let $P^{0}$ be the union of $P$ and the base point. Consider the duality map $T\left(v_{P}\right) \wedge S^{t}\left(P^{0}\right) \rightarrow$ $S^{p+x+t}$ in [52] and [12]. Then the last group in (8-6) is isomorphic to

$\left\{S^{n+\ell+\varkappa} \wedge S^{t}\left(P^{0}\right) ; T\left(\left.\left(\pi_{G_{n+\theta+\varkappa}}^{k}\right) *\left(\hat{\gamma}_{G_{n+\theta+\varkappa}^{\ell}}^{\ell}\right)\right|_{\Omega_{\mathcal{K}}\left(\gamma_{G_{n+\theta+\varkappa}}^{n+\theta+\varkappa}, \mathbb{R}^{p+\theta+\varkappa}\right)}\right) \wedge S^{p+\varkappa+t}\right\}$.

Take a representative map $\alpha$ in a homotopy class in (8-7) and consider the correspondence of a point $y$ in $P$ to a map $\alpha \mid\left(S^{n+\ell+x+t} \wedge\{y\right.$, base point $\left.\}\right)$. It is not difficult to see that the set of $S$-homotopy classes in $(8-7)$ is bijective to the following sets of homotopy classes

$$
\begin{aligned}
& {\left[P, C^{0}\left(S^{n+\ell+\varkappa+t}, T\left(\left.\left(\pi_{G_{n+\theta+\varkappa}}^{k}\right)^{*}\left(\widehat{\gamma}_{G_{n+\theta+\varkappa}^{\ell}}^{\ell}\right)\right|_{\Omega_{\mathcal{K}}\left(\gamma_{G_{n+\theta+\varkappa}}^{n+\theta+\varkappa}, \mathbb{R}^{p+\theta+\varkappa}\right)}\right) \wedge S^{p+\varkappa+t}\right)\right]} \\
& \approx\left[P, C^{0}\left(S^{\ell+n-p}, T\left(\left.\left(\pi_{G_{n+\theta+\varkappa}}^{k}\right)^{*}\left(\hat{\gamma}_{G_{n+\theta+\varkappa}^{\ell}}^{\ell}\right)\right|_{\left.\left.\Omega_{\mathcal{K}}\left(\gamma_{G_{n+\theta+\varkappa}}^{n+\theta+\varkappa}, \mathbb{R}^{p+\theta+\varkappa}\right)\right)\right] .}\right.\right.\right.
\end{aligned}
$$


Setting

$$
B_{\mathfrak{C}}^{\varkappa}=\lim _{\ell \rightarrow \infty} C^{0}\left(S^{\ell+n-p}, T\left(\left.\left(\pi_{G_{n+\theta+\varkappa}}^{k}\right)^{*}\left(\hat{\gamma}_{G_{n+\theta+\varkappa}^{\ell}}^{\ell}\right)\right|_{\Omega_{\mathcal{K}}\left(\gamma_{G_{n+\theta+\varkappa}}^{n+\theta+\varkappa}, \mathbb{R}^{p+\theta+\varkappa}\right)}\right)\right),
$$

we define the classifying space $B_{\mathfrak{C}}=\lim _{\mathcal{H} \rightarrow \infty} B_{\mathfrak{C}}^{\mathcal{L}}$ as in Section 1 . We have the following proposition.

Proposition 8.3 Let $\Omega(n, p)$ be as in Proposition 8.2. Let $P$ be a closed and connected $p$-dimensional manifold such that $P$ is oriented in addition in the oriented case. Then there exists an isomorphism

$$
\begin{aligned}
& \lim _{\varkappa, \ell \rightarrow \infty} \pi_{n+\ell+\varkappa}\left(T\left(\left.\left(\pi_{G_{n+\theta+\varkappa}}^{k}\right)^{*}\left(\hat{\gamma}_{G_{n+\theta+\varkappa}}^{\ell}\right)\right|_{\Omega_{\mathcal{K}}\left(\gamma_{G_{n+\theta+\varkappa}}^{n+\theta+\varkappa}, \mathbb{R}^{p+\theta+\varkappa}\right)}\right) \wedge T\left(v_{P}\right)\right) \\
& \longrightarrow\left[P, B_{\mathfrak{C}}\right],
\end{aligned}
$$

\section{Proof of Theorem 1.3}

The development of the h-principles was described in detail by Gromov [24]. We only refer to the Smale-Hirsch Immersion Theorem [51; 25], the Feit $k$-mersion Theorem [21] and the general theorems due to Gromov [22] and du Plessis [41; 42; 43]. In particular, du Plessis has proposed a nice condition called "extensibility" under which the h-principle holds for $\Omega^{I}$-regular maps or smooth maps with only $\mathcal{K}$-simple singularities. However, this extensibility condition is not so effective in the dimensions $n \geqq p$. On the other hand, Eliashberg [18; 19] proved the famous h-principle on the 1 -jet level for sections $s: N \rightarrow \Omega^{1}(N, P)$ which have a given fold map $f_{0}$ defined around $s^{-1}\left(\Sigma^{1}(N, P)\right)$ such that $\left(j^{2} f_{0}\right)^{-1}\left(\Sigma^{1,0}(N, P)\right)=s^{-1}\left(\Sigma^{1}(N, P)\right)$ and the fold singularities of any semi-index of $f_{0}$ are not empty.

In order to prove Theorem 1.3 by applying Theorems 1.1 and 1.2 we have to show that the assumption concerning h-principles is satisfied in the situation of Theorem 1.3. We proved the h-principle in (h-P) for fold-maps in [3] and [6]. Recently we introduced a very effective condition for the h-principle in (h-P) in [10]. As an application we can prove the following theorem by using [10] (see a proof in [1]). Let $k \gg n, p$, as in Section 1.

Theorem 9.1 Let $n<p$ or $n \geqq p \geqq 2$. Let $\Omega(n, p)$ denote a $\mathcal{K}$-invariant open subspace in $J^{k}(n, p)$ such that when $n \geqq p \geqq 2, \Omega(n, p)$ contains $\Sigma^{n-p+1,0}(n, p)$ at least. Then the h-principle in (h-P) holds for $\Omega(n, p)$.

In particular, we show the following examples of $\Omega(n, p)$ : 
(i) $\Omega^{I}(n, p)$ such that when $n \geqq p \geqq 2, I \geqq(n-p+1,0)$ (see Ando [9]),

(ii) an open subspace consisting of all regular $k$-jets and a finite number of $\mathcal{K}$-orbits of $\mathcal{K}$-simple singularities such that when $n \geqq p \geqq 2$, it contains all fold jets in addition (see Ando [10]).

If $\Omega_{\star}(n+1, p+1)=\Omega_{\mathcal{K}}^{1}$, then we write $\mathfrak{C}(n, P ; \Omega)$ simply in place of $\mathfrak{C}\left(n, P ; \Omega, \Omega_{\mathcal{K}}\right)$ in the following. We have the following corollary of Theorems 1.1, 1.2 and 9.1 and Proposition 7.3.

Corollary 9.2 Let $n<p$. Let $\Omega(n, p)$ and $\Omega_{\mathcal{K}}^{1}$ be the $\mathcal{K}$-invariant open subsets given in Theorem 1.2. Let $P$ be a $p$-dimensional manifold. Then the homomorphism

$$
\omega: \mathfrak{C}(n, P ; \Omega) \longrightarrow \lim _{\ell \rightarrow \infty} \pi_{n+\ell}\left(T\left(\hat{\gamma}_{\mathbf{\Omega}_{\mathcal{K}}^{\ell}}^{\ell}\right)\right) \approx \lim _{\ell \rightarrow \infty} \pi_{n+\ell}\left(T\left(\hat{\gamma}_{\mathbf{\Omega}_{\mathcal{K}}}^{\ell}\right)\right)
$$

is an isomorphism.

If we apply Theorem 9.1 to [45, Theorem 9.2], then under the same assumption of Theorem 1.3, there exists an isomorphism

$$
\mathfrak{C}(n, P ; \Omega) \longrightarrow \lim _{\ell \rightarrow \infty} \pi_{n+\ell}\left(T\left(\hat{\gamma}_{\mathbf{\Omega}_{\mathcal{K}}^{\ell}}^{\ell}\right)\right)
$$

for a sufficiently large integer $m$ also in the dimensions $n \geqq p \geqq 2$ as well as $n<p$. We note that in [45, Theorem 9.2] everything is unoriented, but it works in the oriented case as well. This isomorphism is nothing but the composite of $\omega$ and the homomorphism

$$
\lim _{\ell \rightarrow \infty} \pi_{n+\ell}\left(T\left(\hat{\gamma}_{\mathbf{\Omega}_{\mathcal{K}}^{\ell}}^{1}\right)\right) \longrightarrow \lim _{\ell \rightarrow \infty} \pi_{n+\ell}\left(T\left(\hat{\gamma}_{\mathbf{\Omega}_{\mathcal{K}}^{\ell}}^{\ell}\right)\right)
$$

which is induced by $T\left(\mathbf{b}(\widehat{\gamma})^{\left(\Omega_{\mathcal{K}}^{1}, \Omega_{\mathcal{K}}^{m}\right)}\right)$.

We are now ready to prove Theorem 1.3.

Proof of Theorem 1.3 If $n<p$, then we have

$$
\mathfrak{C}(n, P ; \Omega) \approx \lim _{\ell \rightarrow \infty} \pi_{n+\ell}\left(T\left(\hat{\gamma}_{\mathbf{\Omega}_{\mathcal{K}}^{\ell}}^{\ell}\right)\right)
$$

by Corollary 9.2. If $n \geqq p \geqq 2$, then the homomorphisms

$$
\left(T\left(\mathbf{b}(\hat{\gamma})^{\left(\Omega_{\mathcal{K}}^{\theta+\varkappa}, \Omega_{\mathcal{K}}^{\theta+\varkappa+q}\right)}\right)\right)_{*}: \pi_{n+\ell}\left(T\left(\hat{\gamma}_{\mathbf{\Omega}_{\mathcal{K}} \theta+\varkappa}^{\ell}\right)\right) \longrightarrow \pi_{n+\ell}\left(T\left(\hat{\gamma}_{\boldsymbol{\Omega}_{\mathcal{K}}^{\theta+\varkappa+q}}^{\ell}\right)\right)
$$

are isomorphisms for integers $q \geqq 0$ by Proposition 7.3. By Propositions 8.2 and 8.3 and $(9-1) \mathfrak{C}(n, P ; \Omega)$ is isomorphic to $\left[P, B_{\mathfrak{C}}\right]$. This completes the proof.

The following corollary should be compared with [56, Proposition 88]. 
Corollary 9.3 Under the same assumption of Theorem 1.3, $\mathfrak{O}(n, P ; \Omega) \otimes \mathbb{Q}$ is isomorphic to $H_{n}\left(\Omega_{\mathcal{K}}\left(\gamma_{\widetilde{G}_{n+\theta+\varkappa, \ell}}^{n+\theta+\varkappa}, \mathbb{R}^{p+\theta+\varkappa}\right) \times P ; \mathbb{Q}\right)$ in the dimensions $n<p$ and $n \geqq p \geqq 2$, where $\ell \gg n, p$.

Here, let us see a relationship between the space $B_{\mathfrak{O}}$, the Thom-Atiyah duality in bordism and cobordism, and the spaces introduced by Kazarian $[31 ; 32]$. The unoriented case is not argued but is similar. Let us recall the $n$-dimensional oriented bordism group $\Omega_{n}(P)$ of maps to $P$ and the Thom-Atiyah duality, $\pi_{n+\ell}\left(M S O(\ell) \wedge P^{0}\right) \approx$ $\left[P, \Omega^{\ell} M S O(\ell+p-n)\right]$ (see Atiyah [11] and Conner and Floyd [17, Chapter I, 12]), where $M S O$ denotes the Thom space of the universal bundle $\gamma$ over $B S O$ and $\Omega^{\ell} X$ denotes the $\ell$-th iterated loop space. Setting

$$
\boldsymbol{\Omega}_{\mathcal{K}}^{1}=\Omega_{\mathcal{K}}\left(\gamma_{\widetilde{G}_{n+1, \ell}}^{n+1}, T P \oplus \varepsilon_{P}^{1}\right) \quad \text { and }\left.\quad \boldsymbol{\Omega}_{\mathcal{K}}^{\theta+\varkappa}\right|_{*}=\Omega_{\mathcal{K}}\left(\gamma_{\widetilde{G}_{n+\theta+\varkappa, \ell+p}}^{n+\theta+\varkappa}, \mathbb{R}^{p+\theta+\varkappa}\right),
$$

we have the following commutative diagram $(\ell \gg n, p)$, although we do not give a proof of the commutativity:



Let us explain the homomorphisms in this diagram.

(1) For the left vertical maps, the first vertical map is $\mathfrak{n}^{\left(\Omega, \Omega_{\mathcal{K}}\right)}$. We have the bundle map

$\boldsymbol{b}:\left.\left(\pi_{\boldsymbol{G}_{n+\theta}}^{k}\right)^{*}\left(\hat{\gamma}_{\boldsymbol{G}_{n+\theta+\varkappa}}^{\ell}\right)\right|_{\boldsymbol{\Omega}_{\mathcal{K}}^{\theta}} \oplus \varepsilon_{\boldsymbol{\Omega}_{\mathcal{K}}^{\theta}}^{p+m} \longrightarrow\left(\left.\left(\pi_{\mathbb{G}_{n+\theta+\varkappa}}^{k}\right)^{*}\left(\hat{\gamma}_{\mathbb{G}_{n+\theta+\varkappa}+p}^{\ell+p}\right)\right|_{\left.\boldsymbol{\Omega}_{\mathcal{K}}^{\theta+\varkappa}\right|_{*}}\right) \times \varepsilon_{\boldsymbol{P}}^{m}$ 
covering the composite of the $(n+1)$-homotopy equivalent canonical maps $G_{n+\theta} \rightarrow$ $G_{n+\theta+\varkappa}$ and $G_{n+\theta+\varkappa} \rightarrow \mathbb{G}_{n+\theta+\varkappa}$. It induces the isomorphism

$$
\begin{aligned}
& \pi_{n+\ell}\left(T\left(\left.\left(\pi_{\boldsymbol{G}_{n+\theta}}^{k}\right)^{*}\left(\hat{\gamma}_{\boldsymbol{G}_{n+\theta}^{\ell}}^{\ell}\right)\right|_{\boldsymbol{\Omega}_{\mathcal{K}}^{\theta}}\right)\right) \\
& \approx \pi_{n+\ell+p+m}\left(T\left(\left.\left(\pi_{\boldsymbol{G}_{n+\theta}}^{k}\right)^{*}\left(\hat{\gamma}_{\boldsymbol{G}_{n+\theta}^{\ell}}^{\ell}\right)\right|_{\boldsymbol{\Omega}_{\mathcal{K}}^{\theta}}\right) \wedge S^{p+m}\right) \\
& \stackrel{T(\boldsymbol{b})_{*}}{\longrightarrow} \pi_{n+\ell+p+m}\left(T\left(\left.\left(\pi_{\mathbb{G}_{n+\theta+\varkappa}}^{k}\right)^{*}\left(\hat{\gamma}_{\mathbb{G}_{n+\theta+\varkappa}^{\ell+p}}\right)\right|_{\left.\boldsymbol{\Omega}_{\mathcal{K}}^{\theta+\varkappa}\right|_{*}}\right) \wedge S^{m}\left(P^{0}\right)\right) .
\end{aligned}
$$

The second vertical map is obtained by composing $T\left(\mathbf{b}(\hat{\gamma})^{\left(\Omega_{\mathcal{K}}^{1}, \Omega_{\mathcal{K}}^{\theta}\right)}\right)_{*}$ for fixed $\ell$ as in (9-2) and this isomorphism. The third vertical map is obtained by inserting this isomorphism in the middle of the isomorphism in Proposition 8.2 together with the isomorphism in Proposition 8.3.

(2) The second and the bottom horizontal homomorphisms are induced from the canonical projections

$$
\left.\boldsymbol{\Omega}_{\mathcal{K}}^{\theta+\varkappa}\right|_{*} \subset J^{k}\left(\gamma_{\widetilde{G}_{n+\theta+\varkappa, \ell+p}^{n+\theta+\varkappa}}^{n+\mathbb{R}^{p+\theta+\varkappa}}\right) \longrightarrow \widetilde{G}_{n+\theta+\varkappa, \ell+p} \subset B S O(\ell+p) .
$$

(3) The left vertical map is an isomorphism by Theorems 1.1, 1.2, (9-1), Propositions 8.2 and 8.3 for $n<p$ and $n \geqq p \geqq 2$.

According to [31] and [32, 2.18 Corollary and 2.8 Example], let us consider the subspace $\Omega^{\ell+n-p} M S O(\ell)_{\Omega(n, p)}$ in $\Omega^{\ell+n-p} M S O(\ell)$ which consists of all maps $a: S^{\ell+n-p} \rightarrow M S O(\ell)$ where $a$ is smooth around $a^{-1}(B S O(\ell))$ and for any point $x \in a^{-1}(B S O(\ell))$ with $a(x)=y$, the $k$-jet of the composite of $a:\left(S^{\ell+n-p}, x\right) \rightarrow$ $(M S O(\ell), y)$ and a projection germ of $(M S O(\ell), y)$ to the fiber $\left(\gamma_{y}, y\right)$ lies in $\Omega_{\mathcal{K}}\left(S^{\ell+n-p}, \gamma_{y}\right)$ associated to $\Omega(n, p)$. Then the cobordism class represented by an $\Omega$-regular map is mapped to the homotopy class represented by a map $P \rightarrow$ $\Omega^{\ell+n-p} M S O(\ell)_{\Omega(n, p)}$ in (9-3), whose corresponding map $S^{\ell+n-p} \times P \rightarrow M S O(\ell)$ is transverse to $B S O(\ell)$.

\section{Fold-maps}

Let $m \geqq q$. Let $V_{m, q}^{\text {row }}$ denote the Stiefel manifold $\left(E_{q} \times O(m-q)\right) \backslash O(m)$ under the canonical bases of $\mathbb{R}^{m}$ and $\mathbb{R}^{q}$, whose element is regarded as an epimorphism $\mathbb{R}^{m} \rightarrow \mathbb{R}^{q}$ or a regular $q \times m$-matrix in the following. Let $\mathcal{E} \rightarrow X$ and $\mathcal{F} \rightarrow Y$ be vector bundles of dimensions $m$ and $q$ with metrics respectively. Let $V(\mathcal{E}, \mathcal{F})$ denote the subbundle of $\operatorname{Hom}(\mathcal{E}, \mathcal{F})$ associated to $V_{m, q}^{\text {row }}$.

We have the actions of $O(q) \times O(m)$ on $V_{m+1, q}^{\text {row }}$ from the left-hand side through $O(q)$ and from the right-hand side through $O(m) \times 1$ respectively. The group $O(q) \times O(m)$ 
also naturally acts on $\Omega^{m-q+1,0}(m, q)$. In [5, Theorem 2.6] we described the homotopy type of $\Omega^{m-q+1,0}(m, q)$ in terms of orthogonal groups and Stiefel manifolds, and have given a topological embedding

$$
i_{V, \Omega}=i_{V, \Omega}^{m, q}: V_{m+1, q}^{\mathrm{row}} \rightarrow \Omega^{m-q+1,0}(m, q),
$$

which is equivariant with respect to the actions of $O(q) \times O(m)$. Furthermore, if $m-q+1$ is odd, then there exists an equivariant map

$$
R_{\Omega, V}=R_{\Omega, V}^{m, q}: \Omega^{m-q+1,0}(m, q) \rightarrow V_{m+1, q}^{\text {row }}
$$

such that $R_{\Omega, V} \circ i_{V, \Omega}$ is the identity of $V_{m+1, q}^{\text {row }}$. In particular, we note that if $m=q$, then $i_{V, \Omega} \circ R_{\Omega, V}$ is a deformation retraction of $\Omega^{m-q+1,0}(m, q)$. We note that the image of $i_{V, \Omega}^{m, q}$ is a deformation retract of the open subspace consisting of all regular jets and all definite fold jets in $\Omega^{m-q+1,0}(m, q)$.

If $i_{+1}\left(j_{0}^{2} f\right)$ were defined by $i_{+1}\left(j_{0}^{2} f\right)=j_{0}^{2}\left(\mathrm{id}_{\mathbb{R}} \times f\right)$, then the following technical modification of $i_{V, \Omega}$ and $R_{\Omega, V}$ followed by Lemma 10.1 is unnecessary. Let $h_{t}: \mathbb{R}^{t} \rightarrow$ $\mathbb{R}^{t}$ be the map reversing the order of coordinates as

$$
h_{t}\left(x_{1}, x_{2}, \cdots, x_{t-1}, x_{t}\right)=\left(x_{t}, x_{t-1}, \cdots, x_{2}, x_{1}\right) .
$$

Define

and

$$
\begin{aligned}
\mathfrak{i}_{V, \Omega} & =\mathfrak{i}_{V, \Omega}^{m, q}: V_{m+1, q}^{\text {row }} \rightarrow \Omega^{m-q+1,0}(m, q) \\
\mathfrak{R}_{\Omega, V} & =\mathfrak{R}_{\Omega, V}^{m, q}: \Omega^{m-q+1,0}(m, q) \rightarrow V_{m+1, q}^{\text {row }}
\end{aligned}
$$

by $\mathfrak{i}_{V, \Omega}^{m, q}(A)=i_{V, \Omega}^{m, q}\left(A h_{m+1}\right) \cdot h_{m} \quad$ and $\quad \mathfrak{R}_{\Omega, V}^{m, q}\left(j_{0}^{2} f\right)=R_{\Omega, V}^{m, q}\left(j_{0}^{2}\left(f \circ h_{m}\right)\right) h_{m+1}$.

It will be easy to see

$$
\mathfrak{R}_{\Omega, V}^{m, q} \circ \mathfrak{i}_{V, \Omega}^{m, q}=\mathrm{id}_{V_{m+1, q}^{\text {row }}} .
$$

Let $i^{+1}: J^{k}(m, q) \rightarrow J^{k}(m+1, q+1)$ denote the map defined by $i^{+1}\left(j_{0}^{k} f\right)=$ $j_{0}^{k}\left(\mathrm{id}_{\mathbb{R}} \times f\right)$. Let

$$
j^{+1}, j_{+1}: V_{m+1, q}^{\mathrm{row}} \rightarrow V_{m+2, q+1}^{\mathrm{row}}
$$

denote the map defined by $j^{+1}(A)=(1) \dot{+} A$ and $j_{+1}(A)=A \dot{+}(1)$, where $\dot{+}$ denotes the direct sum of matrices. We consider another action of $O(m)$ on $V_{m+1, q}^{\text {row }}$, distinguished as $O(m)^{*}$, by $T \cdot A=A\left((1) \dot{+} T^{-1}\right)$ for $A \in V_{m+1, q}^{\text {row }}$ and $T \in O(m)$.

Lemma $10.1 \quad$ (i) $\mathfrak{i}_{V, \Omega}^{m, q}$ and $\mathfrak{R}_{\Omega, V}^{m, q}$ are equivariant with respect to the actions of $O(q) \times O(m)$ on $J^{2}(m, q)$ and of $O(q) \times O(m)^{*}$ on $V_{m+1, q}^{\text {row }}$.

(ii) $j_{+1}: V_{m+1, q}^{\text {row }} \rightarrow V_{m+2, q+1}^{\text {row }}$ is equivariant with respect to the actions of $O(q) \times$ $O(m)^{*}$ on $V_{m+1, q}^{\text {row }}$ and on $V_{m+2, q+1}^{\text {row }}$ through $(O(q) \times 1) \times(O(m) \times 1)^{*}$. 
(iii) The following formulas hold:

$$
\begin{aligned}
\mathfrak{R}_{\Omega, V}^{m+1, q+1}\left(i_{+1}\left(j_{0}^{2}(f)\right)\right) & =j_{+1}\left(\Re_{\Omega, V}^{m, q}\left(j_{0}^{2} f\right)\right) \\
\mathfrak{i}_{V, \Omega}^{m+1, q+1}\left(j_{+1}(A)\right) & =i_{+1}\left(\mathfrak{i}_{V, \Omega}^{m, q}(A)\right)
\end{aligned}
$$

Proof Let $A \in V_{m+1, q}^{\text {row }}, S \in O(q)$ and $T \in O(m)$.

(i) We have that

$$
\begin{aligned}
\mathfrak{i}_{V, \Omega}^{m, q}\left(\left(S, T^{-1}\right) \cdot A\right) & =\mathfrak{i}_{V, \Omega}^{m, q}(S A((1) \dot{+} T)) \\
& =i_{V, \Omega}^{m, q}\left(S A((1) \dot{+} T) h_{m+1}\right) \cdot h_{m} \\
& =S \cdot i_{V, \Omega}^{m, q}\left(A h_{m+1} h_{m+1}((1) \dot{+} T) h_{m+1}\right) \cdot h_{m} \\
& =S \cdot i_{V, \Omega}^{m, q}\left(A h_{m+1}\left(h_{m} T h_{m} \dot{+}(1)\right)\right) \cdot h_{m} \\
& =S \cdot i_{V, \Omega}^{m, q}\left(A h_{m+1}\right) \cdot\left(h_{m} T h_{m} h_{m}\right) \\
& =S \cdot\left(i_{V, \Omega}^{m, q}\left(A h_{m+1}\right) \cdot h_{m}\right) \cdot T \\
& =\left(S, T^{-1}\right) \cdot i_{V, \Omega}^{m, q}(A), \\
\mathfrak{R}_{\Omega, V}^{m, q}\left(\left(S, T^{-1}\right) \cdot\left(j_{0}^{2} f\right)\right) & =\Re_{\Omega, V}^{m, q}\left(S \cdot\left(j_{0}^{2} f\right) \cdot T\right) \\
& =R_{\Omega, V}^{m, q}\left(S \cdot\left(j_{0}^{2}(f) \cdot T\right) \cdot h_{m}\right) h_{m+1} \\
& =S R_{\Omega, V}^{m, q}\left(j_{0}^{2}(f) \cdot\left(h_{m} h_{m}\right) T \cdot h_{m}\right) h_{m+1} \\
& =S R_{\Omega, V}^{m, q}\left(j_{0}^{2}(f) \cdot h_{m} \cdot\left(h_{m} T h_{m}\right)\right) h_{m+1} \\
& =S R_{\Omega, V}^{m, q}\left(j_{0}^{2}\left(f \circ h_{m}\right)\right)\left(h_{m} T h_{m} \dot{+}(1)\right) h_{m+1} \\
& =S R_{\Omega, V}^{m, q}\left(j_{0}^{2}\left(f \circ h_{m}\right)\right) h_{m+1} h_{m+1}\left(h_{m} T h_{m} \dot{+}(1)\right) h_{m+1} \\
& =S R_{\Omega, V}^{m, q}\left(j_{0}^{2}\left(f \circ h_{m}\right)\right) h_{m+1}((1) \dot{+} T) \\
& =\left(S, T^{-1}\right) \cdot \Re_{\Omega, V}^{m, q}\left(j_{0}^{2} f\right) .
\end{aligned}
$$

(ii) We have that

$$
\begin{aligned}
j_{+1}\left(\left(S, T^{-1}\right) \cdot A\right) & =S A((1) \dot{+} T) \dot{+}(1) \\
& =(S \dot{+}(1))(A \dot{+}(1))((1) \dot{+} T \dot{+}(1)) \\
& =\left((S \dot{+}(1)),\left(T^{-1} \dot{+}(1)\right)\right) \cdot(A \dot{+}(1)) .
\end{aligned}
$$


(iii) By the definition of $i_{V, \Omega}$ and $R_{\Omega, V}$ we have that

$$
\begin{gathered}
R_{\Omega, V}^{m+1, q+1}\left(j_{0}^{2}\left(\operatorname{id}_{\mathbb{R}} \times f\right)\right)=j^{+1}\left(R_{\Omega, V}^{m, q}\left(j_{0}^{2} f\right)\right), \\
i_{V, \Omega}^{m+1, q+1}\left(j^{+1}(A)\right)=i^{+1}\left(i_{V, \Omega}^{m, q}(A)\right) .
\end{gathered}
$$

Therefore, we have that

$$
\begin{aligned}
\mathfrak{R}_{\Omega, V}^{m+1, q+1}\left(i_{+1}\left(j_{0}^{2}(f)\right)\right) & =R_{\Omega, V}^{m+1, q+1}\left(h_{q+1} h_{q+1} \cdot j_{0}^{2}\left(\left(f \times \mathrm{id}_{\mathbb{R}}\right) \cdot h_{m+1}\right)\right) h_{m+2} \\
& =R_{\Omega, V}^{m+1, q+1}\left(h_{q+1} \cdot j_{0}^{2}\left(\mathrm{id}_{\mathbb{R}} \times\left(h_{q} \circ f \circ h_{m}\right)\right)\right) h_{m+2} \\
& =h_{q+1} j^{+1} R_{\Omega, V}^{m, q}\left(j_{0}^{2}\left(h_{q} \circ f \circ h_{m}\right)\right) h_{m+2} \\
& =\left(h_{q} R_{\Omega, V}^{m, q}\left(j_{0}^{2}\left(h_{q} \circ f \circ h_{m}\right)\right) h_{m+1}\right) \dot{+}(1) \\
& =\left(R_{\Omega, V}^{m, q}\left(j_{0}^{2}\left(f \circ h_{m}\right)\right) h_{m+1}\right) \dot{+}(1) \\
& =j_{+1}\left(\Re_{\Omega, V}^{m, q}\left(j_{0}^{2}(f)\right)\right.
\end{aligned}
$$

and the other formula is similarly proved.

Let $\boldsymbol{\Omega}=\Omega^{m-q+1,0}\left(\gamma_{G_{m}}^{m}, \mathcal{F}\right)$. Let

$$
\begin{array}{r}
i_{V, \Omega}\left(\gamma_{\boldsymbol{G}_{m}}^{m}, \mathcal{F}\right): V\left(\varepsilon_{\boldsymbol{G}_{m}}^{1} \oplus \gamma_{\boldsymbol{G}_{m}}^{m}, \mathcal{F}\right) \rightarrow \boldsymbol{\Omega}, \\
\Re_{\Omega, V}\left(\gamma_{\boldsymbol{G}_{m}}^{m}, \mathcal{F}\right): \boldsymbol{\Omega} \rightarrow V\left(\varepsilon_{\boldsymbol{G}_{m}}^{1} \oplus \gamma_{\boldsymbol{G}_{m}}^{m}, \mathcal{F}\right)
\end{array}
$$

be the fiber maps associated to $\mathfrak{i}_{V, \Omega}$ and $\Re_{\Omega, V}$ respectively. Then

(a) the fiber map $\mathfrak{i}_{V, \Omega}\left(\gamma_{G_{m}}^{m}, \mathcal{F}\right)$ is a topological embedding,

(b) if $m-q+1$ is odd, then $\mathfrak{R}_{\Omega, V}\left(\gamma_{\boldsymbol{G}_{m}}^{m}, \mathcal{F}\right) \circ \mathfrak{i}_{V, \Omega}\left(\gamma_{\boldsymbol{G}_{m}}^{m}, \mathcal{F}\right)$ is the identity of $V\left(\varepsilon_{G_{m}}^{1} \oplus \gamma_{G_{m}}^{m}, \mathcal{F}\right)$.

Let $\hat{\gamma}_{V}^{\ell}$ denote the vector bundle induced from $\hat{\gamma}_{G_{m}}^{\ell}$ over

$$
V\left(\varepsilon_{\boldsymbol{G}_{m}}^{1} \oplus \gamma_{\boldsymbol{G}_{m}}^{m}, \mathcal{F}\right) .
$$

We note $\hat{\gamma}_{\boldsymbol{\Omega}}^{\ell}=\left(\Re_{\Omega, V}\left(\gamma_{G_{m}}^{m}, \mathcal{F}\right)\right)^{*} \hat{\gamma}_{V}^{\ell}$. Then we have the bundle maps

$$
\mathbf{b}_{\mathfrak{R}}: \hat{\gamma}_{\mathbf{\Omega}}^{\ell} \rightarrow \hat{\gamma}_{V}^{\ell} \quad \text { and } \quad \mathbf{b}_{i}: \hat{\gamma}_{V}^{\ell} \rightarrow \hat{\gamma}_{\mathbf{\Omega}}^{\ell}
$$

covering $\Re_{\Omega, V}\left(\gamma_{G_{m}}^{m}, \mathcal{F}\right)$ and $\mathfrak{i}_{V, \Omega}\left(\gamma_{G_{m}}^{m}, \mathcal{F}\right)$ respectively. The associated maps $T\left(\mathbf{b}_{\mathfrak{R}}\right)$ and $T\left(\mathbf{b}_{\mathfrak{i}}\right)$ between the Thom spaces satisfy that $T\left(\mathbf{b}_{\mathfrak{R}}\right) \circ T\left(\mathbf{b}_{\mathfrak{i}}\right)$ is equal to the identity of $T\left(\hat{\gamma}_{V}^{\ell}\right)$. 
We set

$$
\begin{aligned}
\boldsymbol{\Omega} & =\Omega^{n-p+1,0}\left(\gamma_{G_{n}}^{n}, T P\right), \\
\boldsymbol{\Omega}^{\oplus} & =\Omega^{n-p+1,0}\left(\gamma_{G_{n}}^{n} \oplus \varepsilon_{G_{n}}^{1}, T P \oplus \varepsilon_{P}^{1}\right), \\
\boldsymbol{\Omega}_{\mathcal{K}} & =\Omega^{n-p+1,0}\left(\gamma_{G_{n+1}}^{n+1}, T P \oplus \varepsilon_{P}^{1}\right), \\
V_{n+1} & =V\left(\varepsilon_{G_{n}}^{1} \oplus \gamma_{G_{n}}^{n}, T P\right), \\
V_{n+1}^{\oplus} & =V\left(\varepsilon_{G_{n}}^{1} \oplus \gamma_{G_{n}}^{n} \oplus \varepsilon_{G_{n}}^{1}, T P \oplus \varepsilon_{P}^{1}\right), \\
V_{n+2} & =V\left(\varepsilon_{G_{n+1}}^{1} \oplus \gamma_{G_{n+1}}^{n+1}, T P \oplus \varepsilon_{P}^{1}\right) .
\end{aligned}
$$

Let $\Delta^{\oplus}: \boldsymbol{\Omega} \rightarrow \boldsymbol{\Omega}^{\oplus}$ and $\mathbf{v}_{+1}: V_{n+1} \rightarrow V_{n+1}^{\oplus}$ denote the fiber maps associated to $i_{+} \mid \Omega^{n-p+1,0}(n, p)$ and the map $j_{+1}^{n, p}: V_{n+1, p}^{\text {row }} \rightarrow V_{n+2, p+1}^{\text {row }}$ sending $A$ to $A \dot{+}(1)$ respectively. The canonical bundle map

$$
\gamma_{G_{n}}^{n} \oplus \varepsilon_{G_{n}}^{1} \rightarrow \gamma_{G_{n+1}}^{n+1}
$$

covering ${ }_{i}^{G}: G_{n} \rightarrow G_{n+1}$ induces the fiber maps $\mathbf{j}_{\Omega}: \boldsymbol{\Omega}^{\oplus} \rightarrow \boldsymbol{\Omega}_{\mathcal{K}}$ and $\mathbf{j}_{V}: V_{n+1}^{\oplus} \rightarrow$ $V_{n+2}$ covering $i^{G} \times \operatorname{id}_{P}$. Note that $\Delta^{\left(\Omega, \Omega_{\mathcal{K}}\right)}=\mathbf{j}_{\Omega} \circ \Delta^{\oplus}$. Let $n-p+1$ be odd. By Lemma 10.1 we have the commutative diagram:

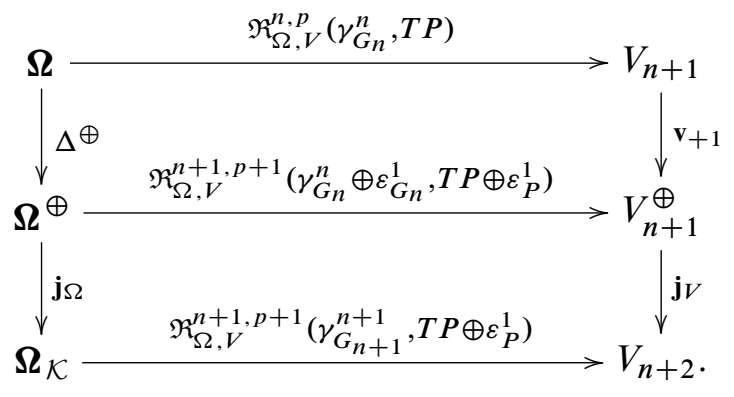

Since $j_{+1}^{n, p}$ and $i^{G} \times \operatorname{id}_{P}$ are homotopy $n$-equivalences, we have the following lemma.

Lemma 10.2 Let $n-p+1$ be odd. The fiber maps $\mathbf{v}_{+1}$ and $\mathbf{j}_{V}$ are homotopy $n$-equivalences.

We have the bundle map $\mathbf{b}_{V}: \hat{\gamma}_{V_{n+1}}^{\ell} \rightarrow \hat{\gamma}_{V_{n+2}}^{\ell}$ covering $\mathbf{j} V \circ \mathbf{v}_{+1}$ and its associated map $T\left(\mathbf{b}_{\mathbf{V}}\right)$ between the Thom spaces. Recalling the map

$$
T\left(\mathbf{b}(\hat{\gamma})^{\left(\Omega, \Omega_{\mathcal{K}}\right)}\right): T\left(\hat{\gamma}_{\boldsymbol{\Omega}}^{\ell}\right) \longrightarrow T\left(\hat{\gamma}_{\mathbf{\Omega}_{\mathcal{K}}}^{\ell}\right)
$$


we obtain the following commutative diagram

$(10-1)$

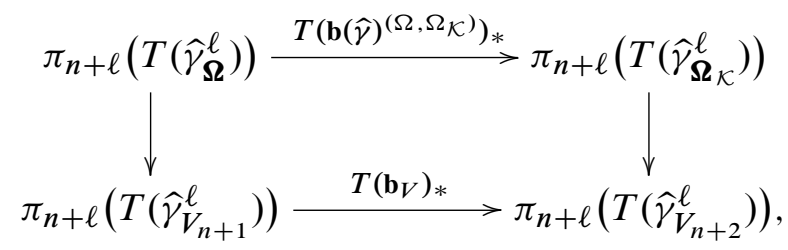

where the left vertical map and the right vertical map are $T\left(\mathbf{b}_{\mathfrak{R}}\right)_{*}$ for $(n, p)$ and $(n+1, p+1)$ respectively.

Proposition 10.3 Let $n \geqq p \geqq 2$ and $\ell \gg n, p$. Let $n-p+1$ be odd. Let $\boldsymbol{\Omega}=$ $\Omega^{n-p+1,0}\left(\gamma_{G_{n}}^{n}, T P\right)$ and $\boldsymbol{\Omega}_{\mathcal{K}}=\Omega^{n-p+1,0}\left(\gamma_{G_{n+1}+1}^{n+1}, T P \oplus \varepsilon_{P}^{1}\right)$ be as above. Then there exist homomorphisms

$$
\begin{gathered}
\omega_{V}: \mathfrak{C}\left(n, P ; \Omega^{n-p+1,0}\right) \longrightarrow \pi_{n+\ell}\left(T\left(\hat{\gamma}_{V_{n+2}}^{\ell}\right)\right), \\
\iota_{V}: \pi_{n+\ell}\left(T\left(\hat{\gamma}_{V_{n+2}}^{\ell}\right)\right) \longrightarrow \mathfrak{C}\left(n, P ; \Omega^{n-p+1,0}\right)
\end{gathered}
$$

such that $\omega_{V} \circ \iota_{V}$ is the identity of $\pi_{n+\ell}\left(T\left(\hat{\gamma}_{V_{n+2}}^{\ell}\right)\right)$. In particular, if $n=p$, then $\omega_{V}$ is an isomorphism.

Proof In the diagram (10-1) it follows from the similar argument as in proof of Theorem 1.2 that $T\left(\mathbf{b}_{V}\right)_{*}$ is an epimorphism. It follows from (a) and (b) that $T\left(\mathbf{b}_{\mathfrak{R}}\right)_{*}$ in the diagram (10-1) for $(n, p)$ and $(n+1, p+1)$ are epimorphisms. By Theorem 1.1 we obtain the required homomorphisms $\omega_{V}$ and $\iota_{V}$.

Here, we prove the following refinement of [5, Theorem 0.3]. This theorem should be compared with the results of Kalmár [27; 28].

Theorem 10.4 Under the assumption of Proposition 10.3 there exists a splitting epimorphism

$$
\mathfrak{C}\left(n, P ; \Omega^{n-p+1,0}\right) \rightarrow \lim _{\varkappa \rightarrow \infty}\left(\lim _{\ell \rightarrow \infty} \pi_{n+\ell+\varkappa}\left(T\left(\hat{\gamma}_{G_{n-p+1}^{\ell}}\right) \wedge T\left(v_{P}\right)\right)\right),
$$

where $P$ is oriented in the oriented case. In particular, if $n=p$, then this is an isomorphism.

Proof The proof proceeds similarly as in the proof of Propositions 8.2 and 8.3 by replacing spaces $\Omega_{\mathcal{K}}(\xi, \mathcal{F})$ by $V(\xi, \mathcal{F})$. The canonical projections are denoted by $\pi_{X}^{V}: V(\mathcal{E}, \mathcal{F}) \rightarrow X$ and $\pi_{Y}^{V}: V(\mathcal{E}, \mathcal{F}) \rightarrow Y$ respectively. We only prove the unoriented case. Define the fiber map

$$
V_{n+2} \longrightarrow V^{\prime}\left(\varepsilon_{G_{n+1}}^{1} \oplus \gamma_{G_{n+1}}^{n+1} \oplus v_{P}, T P \oplus \varepsilon_{P}^{1} \oplus v_{P}\right),
$$


where the last space is the subbundle of

$$
\operatorname{Hom}\left(\pi_{G_{n+1}}^{*}\left(\varepsilon_{G_{n+1}}^{1} \oplus \gamma_{G_{n+1}}^{n+1}\right) \oplus \pi_{P}^{*}\left(v_{P}\right), T P \oplus \varepsilon_{P}^{1} \oplus v_{P}\right)
$$

associated to $V_{n+2+\varkappa, p+1+\varkappa}$ over $G_{n+1} \times P$ and the map sends $A \in\left(V_{n+2}\right)_{(x, y)}$ to $A \oplus \operatorname{id}_{\left(v_{P}\right)_{y}}$. Let $G_{n+2+\varkappa}=A_{n+2+\varkappa, \ell}$ as above. We have a bundle map

$$
\pi_{G_{n+1}}^{*}\left(\varepsilon_{G_{n+1}}^{1} \oplus \gamma_{G_{n+1}}^{n+1}\right) \oplus \pi_{P}^{*}\left(v_{P}\right) \longrightarrow \gamma_{G_{n+2+\varkappa}}^{n+2+\varkappa}
$$

covering a classifying map $c^{+}: G_{n+1} \times P \rightarrow G_{n+2+\varkappa}$ (we may need to replace $\ell$ with a bigger integer). We note that $\left(c^{+}, \pi_{P}\right): G_{n+1} \times P \rightarrow G_{n+2+\varkappa} \times P$ is a homotopy $(n+1)$-equivalence. By (10-2) and (10-3) we obtain a homotopy $(n+1)$-equivalent fiber map

$$
V_{n+2} \longrightarrow V\left(\gamma_{G_{n+2+\varkappa}}^{n+2+\varkappa}, T P \oplus \varepsilon_{P}^{1} \oplus v_{P}\right)
$$

covering $\left(c^{+}, \pi_{P}\right)$. The trivialization $T P \oplus v_{P} \rightarrow \varepsilon_{P}^{p+\varkappa}$ induces the bundle map

$$
V\left(\gamma_{G_{n+2+\varkappa}}^{n+2+\varkappa}, T P \oplus \varepsilon_{P}^{1} \oplus v_{P}\right) \longrightarrow V\left(\gamma_{G_{n+2+\varkappa}}^{n+2+\varkappa}, \mathbb{R}^{p+1+\varkappa}\right) \times P
$$

over $G_{n+2+\varkappa} \times P$.

We denote, by $V_{n-p+1, p+1+\varkappa, \ell}$, the space which consists of all triples $(a, b, c)$ where $a, b$ and $c$ are mutually perpendicular subspaces in $\mathbb{R}^{n+\ell+\varkappa+2}$ of dimensions $n-p+1$, $p+1+\varkappa$ and $\ell$ respectively with $a \oplus b \oplus c=\mathbb{R}^{n+\ell+\varkappa+2}$. Let $\gamma_{V}^{p+1+\varkappa}$ be the canonical vector bundle over $V_{n-p+1, p+1+\varkappa, \ell}$ of dimension $p+1+\varkappa$.

We denote an element of $V\left(\gamma_{G_{n+2+\varkappa}}^{n+2+\varkappa}, \mathbb{R}^{p+1+\varkappa}\right)$ by $(\alpha, h)$, where $\alpha \in G_{n+2+\varkappa}$ and $h \in V\left(\left(\gamma_{G_{n+2+\varkappa}+2+\varkappa}^{n}\right)_{\alpha}, \mathbb{R}^{p+1+\varkappa}\right)$, which is regarded as an epimorphism. Then $(\alpha, h)$ defines $\left(\operatorname{Ker}(h), \operatorname{Ker}(h)^{\perp}, \alpha^{\perp}\right)$ in $V_{n-p+1, p+1+\varkappa, \ell}$, which is the triple of the kernel of $h$, the orthogonal complement of $\operatorname{Ker}(h)$ in $\alpha$ and the orthogonal complement $\alpha^{\perp}$. Let $V\left(\gamma_{V}^{p+1+\varkappa}, \mathbb{R}^{p+1+\varkappa}\right)$ denote the principal bundle with fiber $O(p+1+\varkappa)$ associated to $\operatorname{Hom}\left(\gamma_{V}^{p+1+\varkappa}, \mathbb{R}^{p+1+\varkappa}\right)$. We have the canonical homeomorphism

$$
V\left(\gamma_{G_{n+2+\varkappa}}^{n+2+\varkappa}, \mathbb{R}^{p+1+\varkappa}\right) \rightarrow V\left(\gamma_{V}^{p+1+\varkappa}, \mathbb{R}^{p+1+\varkappa}\right)
$$

which maps $(\alpha, h)$ to $h \mid \operatorname{Ker}(h)^{\perp}: \operatorname{Ker}(h)^{\perp} \rightarrow \mathbb{R}^{p+1+\varkappa}$ over $\left(\operatorname{Ker}(h), \operatorname{Ker}(h)^{\perp}, \alpha^{\perp}\right)$. Let $\mu$ denote the map

$$
\mu: V\left(\gamma_{G_{n+2+\varkappa}}^{n+2+\varkappa}, \mathbb{R}^{p+1+\varkappa}\right)=V\left(\gamma_{V}^{p+1+\varkappa}, \mathbb{R}^{p+1+\varkappa}\right) \longrightarrow V_{n-p+1, p+1+\varkappa, \ell}
$$

defined by $\mu((\alpha, h))=\left(\operatorname{Ker}(h), \operatorname{Ker}(h)^{\perp}, \alpha^{\perp}\right)$. Let

$$
\rho: V_{n-p+1, p+1+\varkappa, \ell} \rightarrow G_{n-p+1, \ell+p+1+\varkappa}
$$

be the map defined by $\rho(a, b, c)=a$. Then $\rho \circ \mu$ gives a fiber bundle. Since $\rho^{-1}\left(\mathbb{R}^{n-p+1} \times 0\right)$ is $G_{p+1+\varkappa, \ell}$ for $0 \times \mathbb{R}^{\ell+p+1+\varkappa}$, we have that $(\rho \circ \mu)^{-1}\left(\mathbb{R}^{n-p+1} \times\right.$ 
$0)$ is $V_{\ell+p+1+\varkappa, p+1+\varkappa}$, namely $O(\ell+p+1+\varkappa) / O(\ell)$. Hence, $\rho \circ \mu$ is a homotopy $\ell$-equivalence. We note that

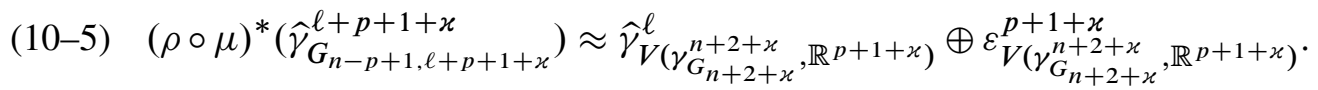
Now $\pi_{n+\ell}\left(T\left(\hat{\gamma}_{V_{n+2}}^{\ell}\right)\right)$ is isomorphic to $\pi_{n+\ell+p+\varkappa}\left(T\left(\hat{\gamma}_{V_{n+2}}^{\ell} \oplus \varepsilon_{V_{n+2}}^{p+\varkappa}\right)\right)$. This is isomorphic to

$$
\pi_{n+\ell+p+\varkappa}\left(T\left(\left.\left(\hat{\gamma}_{V_{n+2}}^{\ell} \oplus\left(\pi_{P}^{V}\right)^{*}\left(T P \oplus v_{P}\right)\right)\right|_{V\left(\gamma_{G_{n+2+\varkappa}}^{n+2+\varkappa}, T P \oplus \varepsilon_{P}^{1} \oplus v_{P}\right)}\right)\right) .
$$

Since setting $G=G_{n+2+\varkappa, \ell+p}$, we have the bundle map

$$
\left.\hat{\gamma}_{V_{n+2}}^{\ell} \oplus\left(\pi_{P}^{V}\right)^{*}\left(T P \oplus v_{P}\right) \longrightarrow\left(\pi_{G}^{V}\right)^{*}\left(\hat{\gamma}_{G}^{\ell+p}\right)\right|_{V\left(\gamma_{G}^{n+2+\varkappa}, \mathbb{R}^{p+1+\varkappa}\right)} \times v_{P}
$$

covering the canonical homotopy $(n+1+\varkappa)$-equivalent map

$$
V\left(\gamma_{G_{n+2+\varkappa}}^{n+2+\varkappa}, T P \oplus \varepsilon_{P}^{1} \oplus v_{P}\right) \longrightarrow V\left(\gamma_{G}^{n+2+\varkappa}, \mathbb{R}^{p+1+\varkappa}\right) \times P,
$$

by (10-4), the last group is isomorphic to

$$
\pi_{n+\ell+p+\varkappa}\left(T\left(\left.\left(\pi_{G}^{V}\right)^{*} \hat{\gamma}_{G}^{\ell+p}\right|_{V\left(\gamma_{G}^{n+2+\varkappa}, \mathbb{R}^{p+1+\varkappa}\right)}\right) \wedge T\left(v_{P}\right)\right) .
$$

This is isomorphic to

$$
\pi_{n+\ell+p+1+2 \varkappa}\left(T\left(\left.\left(\pi_{G}^{V}\right)^{*}\left(\hat{\gamma}_{G}^{\ell+p} \oplus \varepsilon_{G}^{1+\varkappa}\right)\right|_{V\left(\gamma_{G}^{n+2+\varkappa}, \mathbb{R}^{p+1+\varkappa}\right)}\right) \wedge T\left(v_{P}\right)\right) .
$$

Since we have the bundle map of $\hat{\gamma}_{G_{n+2+\varkappa}}^{\ell} \oplus \varepsilon_{G_{n+2+\varkappa}}^{p+1+\varkappa}$ to $\hat{\gamma}_{G}^{\ell+p} \oplus \varepsilon_{G}^{1+\varkappa}$ covering the canonical map $G_{n+2+\varkappa} \rightarrow G$ again, this is isomorphic to

$$
\pi_{n+\ell+p+1+2 \varkappa}\left(T\left(\left.\left(\pi_{G_{n+2+\varkappa}}^{V}\right)^{*}\left(\hat{\gamma}_{G_{n+2+\varkappa}}^{\ell} \oplus \varepsilon_{G_{n+2+\varkappa}}^{p+1+\varkappa}\right)\right|_{V\left(\gamma_{G_{n+2+\varkappa}}^{n+2+\varkappa}, \mathbb{R}^{p+1+\varkappa}\right)}\right) \wedge T\left(v_{P}\right)\right) \text {. }
$$

Since $\rho \circ \mu$ is a homotopy $\ell$-equivalence, it follows from (10-5) that the last group is isomorphic to

$$
\pi_{n+\ell+p+1+2 \varkappa}\left(T\left(\hat{\gamma}_{G_{n-p+1, \ell+p+1+\varkappa}^{\ell+p+1+\varkappa}}\right) \wedge T\left(v_{P}\right)\right),
$$

which is isomorphic to

$$
\pi_{n+\ell+\varkappa}\left(T\left(\hat{\gamma}_{G_{n-p+1}}^{\ell}\right) \wedge T\left(v_{P}\right)\right) \approx\left\{S^{n+\ell+\varkappa} ; T\left(\hat{\gamma}_{G_{n-p+1}}^{\ell}\right) \wedge T\left(v_{P}\right)\right\} .
$$

Since $\ell$ is sufficiently large and $\varkappa$ is any integer with $\varkappa \geqq p+3$, we have proved the assertion.

Now we define

$$
B_{V}=\lim _{\ell \rightarrow \infty} C^{0}\left(S^{n-p+\ell}, T\left(\hat{\gamma}_{G_{n-p+1}}^{\ell}\right)\right)
$$


By the duality map $T\left(v_{P}\right) \wedge S^{t}\left(P^{0}\right) \rightarrow S^{p+\varkappa+t}$, the last group in (10-6) is isomorphic to

$$
\left\{S^{n+\ell+\varkappa} \wedge S^{t}\left(P^{0}\right) ; T\left(\hat{\gamma}_{G_{n-p+1}}^{\ell}\right) \wedge S^{p+\varkappa+t}\right\} \approx\left\{S^{n-p+\ell} \wedge P^{0} ; T\left(\hat{\gamma}_{G_{n-p+1}}^{\ell}\right)\right\} .
$$

Then we have the following proposition.

Proposition 10.5 Let $P$ be a closed and connected $p$-dimensional manifold such that $P$ is oriented in addition in the oriented case. Then we have the isomorphism

$$
\lim _{\ell \rightarrow \infty} \pi_{n+\ell}\left(T\left(\hat{\gamma}_{V_{n+2}}^{\ell}\right)\right) \longrightarrow\left[P, B_{V}\right]
$$

where $V_{n+2}=V\left(\varepsilon_{G_{n+1}}^{1} \oplus \gamma_{G_{n+1}}^{n+1}, T P \oplus \varepsilon_{P}^{1}\right)$.

Furthermore, let $n=p$ and $F$ be the space defined in Section 1. It was proved in [5, Proposition 4.1 and Remark 4.3] and [3] by applying Theorem 10.4 that if $P$ is closed, connected and oriented, then there exists an isomorphism

$$
\mathfrak{O}\left(n, P ; \Omega^{1,0}\right) \approx[P, F] .
$$

In particular, the homotopy group $\pi_{n}(F, *)$ of the $F$ 's connected component of maps of degree 0 is isomorphic to the $n$-th stable homotopy group $\pi_{n}^{s}$ by [3, Theorem 1 and Corollary 2].

Chess [16, 1.3 Corollary] proved, in our notation, that $\mathfrak{O}\left(n, \mathbb{R}^{n} ; \Omega^{1,0}\right)$ is isomorphic to $\pi_{n}^{s}$. We can prove this fact from (10-7). In fact, let $\mathcal{O}\left(n, S^{n} ; \Omega^{1,0} ; 0\right)$ denote the subset of $\mathfrak{O}\left(n, S^{n} ; \Omega^{1,0}\right)$ which consists of all cobordism classes $[f]$ such that the degree of $f$ is 0 . By applying the h-principles in (h-P) for fold-maps to $\mathbb{R}^{n}$ and $S^{n}$ in [6] we can prove that the inclusion $\mathbb{R}^{n}=S^{n} \backslash\{(0, \cdots, 0,1)\} \rightarrow S^{n}$ canonically induces an isomorphism

$$
\mathfrak{O}\left(n, \mathbb{R}^{n} ; \Omega^{1,0}\right) \rightarrow \mathfrak{O}\left(n, S^{n} ; \Omega^{1,0} ; 0\right) .
$$

The detail is left to the reader.

The author proposes a problem: Let $\mathfrak{i}_{\Omega}: \pi_{n}^{s} \approx \mathfrak{O}\left(n, S^{n} ; \Omega^{1,0} ; 0\right) \rightarrow \mathfrak{O}\left(n, S^{n} ; \Omega\right)$ denote the homomorphism induced from the inclusion $\Omega^{1,0}(n, n) \rightarrow \Omega(n, n)$. For an element $a \neq 0$ in $\pi_{n}^{s}$, we define a $\mathcal{K}$-invariant open subset $U(a)$ in $J^{k}(n, n)$ as the union of all $\mathcal{K}$-invariant open sets $\Omega(n, n)$ such that $i_{\Omega}(a) \neq 0$. Study how the singularities in $U(a)$ are related to $a$. By the definition of $\mathfrak{i}_{V, \Omega}$, Propositions 10.3 and 10.5 , Theorem 10.4 and [5, Remark 4.3], the homomorphism $\mathfrak{O}\left(n, S^{n} ; \Omega^{1,0}\right) \rightarrow \mathfrak{O}\left(n, S^{n} ; \Omega\right)$ corresponds to

$$
\left[S^{n}, F\right] \longrightarrow\left[S^{n}, B_{\mathfrak{O}}\right] \text { induced from } F \longrightarrow B_{\mathfrak{O}}
$$


which is constructed from the inclusion of a point to the space

$$
\Omega_{\mathcal{K}}\left(\gamma_{\widetilde{G}_{n+m, \ell}}^{n+m}, \mathbb{R}^{p+m}\right) .
$$

The spaces $B_{\mathfrak{O}}$ 's for $\mathfrak{O}\left(n, S^{n} ; \Omega\right)$ will be useful. This should be compared with the theme and the problem studied in [8].

Acknowledgements The author would like to express his gratitude to Professor O Saeki for helpful discussions and comments. The author would like to thank the referee for his/her kind and helpful suggestions.

\section{References}

[1] Y Ando, Smooth maps with singularities of bounded $\mathcal{K}$-codimensions arXiv: math.GT/0704.0115

[2] Y Ando, The homotopy type of the space consisting of regular jets and folding jets in $J^{2}(n, n)$, Japan. J. Math. (N.S.) 24 (1998) 169-181 MR1630137

[3] Y Ando, Fold-maps and the space of base point preserving maps of spheres, J. Math. Kyoto Univ. 41 (2001) 693-737 MR1891672

[4] Y Ando, Folding maps and the surgery theory on manifolds, J. Math. Soc. Japan 53 (2001) 357-382 MR1815139

[5] Y Ando, Invariants of fold-maps via stable homotopy groups, Publ. Res. Inst. Math. Sci. 38 (2002) 397-450 MR1903746

[6] Y Ando, Existence theorems of fold-maps, Japan. J. Math. (N.S.) 30 (2004) 29-73 MR2070370

[7] Y Ando, Smooth maps having only singularities with Boardman symbol $(1,0)$, Topology Appl. 142 (2004) 205-226 MR2071303

[8] Y Ando, Stable homotopy groups of spheres and higher singularities, J. Math. Kyoto Univ. 46 (2006) 147-165 MR2260821

[9] Y Ando, A homotopy principle for maps with prescribed Thom-Boardman singularities, Trans. Amer. Math. Soc. 359 (2007) 489-515 MR2255183

[10] Y Ando, The homotopy principle for maps with singularities of given $\mathcal{K}$-invariant class, J. Math. Soc. Japan 59 (2007) 557-582 MR2326177

[11] M F Atiyah, Bordism and cobordism, Proc. Cambridge Philos. Soc. 57 (1961) 200-208 MR0126856

[12] MF Atiyah, Thom complexes, Proc. London Math. Soc. (3) 11 (1961) 291-310 MR0131880

[13] J M Boardman, Singularities of differentiable maps, Inst. Hautes Études Sci. Publ. Math. (1967) 21-57 MR0231390 
[14] O Burlet, G de Rham, Sur certaines applications génériques d'une variété close à 3 dimensions dans le plan, Enseignement Math. (2) 20 (1974) 275-292 MR0365594

[15] H Cartan, S Eilenberg, Homological algebra, Princeton University Press (1956) MR0077480

[16] D S Chess, Singularity theory and configuration space models of $\Omega^{n} S^{n}$ of nonconnected spaces, Topology Appl. 25 (1987) 313-338 MR889874

[17] PE Conner, E E Floyd, Differentiable periodic maps, Ergebnisse der Math. und ihrer Grenzgebiete, N. F. 33, Academic Press, New York (1964) MR0176478

[18] J Eliashberg, Singularities of folding type, Izv. Akad. Nauk SSSR Ser. Mat. 34 (1970) 1110-1126 MR0278321

[19] J Eliashberg, Surgery of singularities of smooth mappings, Izv. Akad. Nauk SSSR Ser. Mat. 36 (1972) 1321-1347 MR0339261

[20] J Eliashberg, Cobordisme des solutions de relations différentielles, from: "South Rhone seminar on geometry, I (Lyon, 1983)", Travaux en Cours, Hermann, Paris (1984) 17-31 MR753850

[21] S D Feit, $k$-mersions of manifolds, Acta Math. 122 (1969) 173-195 MR0243541

[22] M L Gromov, Stable mappings of foliations into manifolds, Izv. Akad. Nauk SSSR Ser. Mat. 33 (1969) 707-734 MR0263103

[23] M L Gromov, A topological technique for the construction of solutions of differential equations and inequalities, from: "Actes du Congrès International des Mathématiciens (Nice, 1970), Tome 2", Gauthier-Villars, Paris (1971) 221-225 MR0420697

[24] M L Gromov, Partial differential relations, Ergebnisse der Math. und ihrer Grenzgebiete [Results in Math. and Related Areas] (3) 9, Springer, Berlin (1986) MR864505

[25] M W Hirsch, Immersions of manifolds, Trans. Amer. Math. Soc. 93 (1959) 242-276 MR0119214

[26] K Ikegami, O Saeki, Cobordism group of Morse functions on surfaces, J. Math. Soc. Japan 55 (2003) 1081-1094 MR2003761

[27] B Kalmár, Cobordism invariants of fold maps arXiv:0709.0350

[28] B Kalmár, Cobordism of fold maps, stably framed manifolds and immersions arXiv: 0803.1666

[29] B Kalmár, Cobordism group of Morse functions on unoriented surfaces, Kyushu J. Math. 59 (2005) 351-363 MR2188598

[30] B Kalmár, Cobordism group of fold maps of oriented 3-manifolds into the plane, Acta Math. Hungar. 117 (2007) 1-25 MR2356183

[31] M Kazarian, Multisingularities, cobordisms, and enumerative geometry, Uspekhi Mat. Nauk 58 (2003) 29-88 MR2042262 
[32] M Kazarian, Thom polynomials, from: "Singularity theory and its applications", Adv. Stud. Pure Math. 43, Math. Soc. Japan, Tokyo (2006) 85-135 MR2313410

[33] S Kobayashi, K Nomizu, Foundations of differential geometry. Vol I, Interscience Publishers John Wiley \& Sons, New York-London (1963) MR0152974

[34] J A Lees, On the classification of Lagrange immersions, Duke Math. J. 43 (1976) 217-224 MR0410764

[35] H I Levine, Singularities of differentiable maps, from: "Proceedings of Liverpool Singularities Symposium, (1969/1970)”, Lecture Notes in Math. 192, Springer, Berlin (1971) 1-85

[36] J Martinet, Déploiements versels des applications différentiables et classification des applications stables, from: "Singularités d'applications différentiables (Sém., Plans-surBex, 1975)", Lecture Notes in Math. 535, Springer, Berlin (1976) 1-44 MR0649264

[37] J N Mather, Stability of $C^{\infty}$ mappings. III. Finitely determined mapgerms, Inst. Hautes Études Sci. Publ. Math. (1968) 279-308 MR0275459

[38] J N Mather, Stability of $C^{\infty}$ mappings. IV. Classification of stable germs by $R-$ algebras, Inst. Hautes Études Sci. Publ. Math. (1969) 223-248 MR0275460

[39] J N Mather, On Thom-Boardman singularities, from: "Dynamical systems (Proc. Sympos., Univ. Bahia, Salvador, 1971)”, Academic Press, New York (1973) 233-248 MR0353359

[40] A Phillips, Submersions of open manifolds, Topology 6 (1967) 171-206 MR0208611

[41] A du Plessis, Maps without certain singularities, Comment. Math. Helv. 50 (1975) 363-382 MR0397779

[42] A du Plessis, Contact-Invariant regularity conditions, from: "Singularités d'applications différentiables (Sém., Plans-sur-Bex, 1975)", Lecture Notes in Math. 535, Springer, Berlin (1976) 205-236 MR0482811

[43] A du Plessis, Homotopy classification of regular sections, Compositio Math. 32 (1976) 301-333 MR0413163

[44] R Rimányi, A Szücs, Pontrjagin-Thom-type construction for maps with singularities, Topology 37 (1998) 1177-1191 MR1632908

[45] R Sadykov, Bordism groups of solutions to differential relations arXiv: math.AT/0608460

[46] R Sadykov, Bordism groups of special generic mappings, Proc. Amer. Math. Soc. 133 (2005) 931-936 MR2113946

[47] O Saeki, Topology of special generic maps into $\mathbf{R}^{3}$, Mat. Contemp. 5 (1993) 161-186 MR1305180 Workshop on Real and Complex Singularities (São Carlos, 1992)

[48] O Saeki, Topology of singular fibers of differentiable maps, Lecture Notes in Math. 1854, Springer, Berlin (2004) MR2106689 
[49] O Saeki, K Sakuma, On special generic maps into $\mathbf{R}^{3}$, Pacific J. Math. 184 (1998) 175-193 MR1626540

[50] J-P Serre, Groupes d'homotopie et classes de groupes abéliens, Ann. of Math. (2) 58 (1953) 258-294 MR0059548

[51] S Smale, The classification of immersions of spheres in Euclidean spaces, Ann. of Math. (2) 69 (1959) 327-344 MR0105117

[52] E H Spanier, Function spaces and duality, Ann. of Math. (2) 70 (1959) 338-378 MR0107862

[53] E H Spanier, Algebraic topology, McGraw-Hill Book Co., New York (1966) MR0210112

[54] N Steenrod, The topology of fibre bundles, Princeton Math. Series 14, Princeton University Press (1951) MR0039258

[55] R E Stong, Notes on cobordism theory, Math. notes, Princeton University Press (1968) MR0248858

[56] A Szúcs, Cobordism of singular maps, Geom. Topol. (2008) 2379-2452

[57] R Thom, Quelques propriétés globales des variétés différentiables, Comment. Math. Helv. 28 (1954) 17-86 MR0061823

Department of Mathematical Sciences, Faculty of Science, Yamaguchi University Yamaguchi 753-8512, Japan

andoy@yamaguchi-u.ac.jp

Received: 6 June 2006 Revised: 18 August 2008 